\title{
Fast and Slow Paths to Quiescence: Ages and Sizes of 400 Quiescent Galaxies from the LEGA-C Survey
}

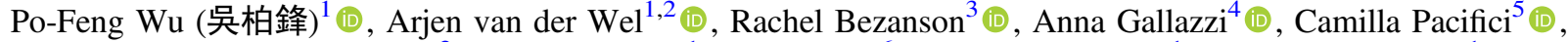 \\ Caroline M. S. Straatman ${ }^{2}$ (i), Ivana Barišicic ${ }^{1}$, Eric F. Bell ${ }^{6}$ (i), Priscilla Chauke ${ }^{1}$ (i), Josha van Houdt ${ }^{1}$, \\ Marijn Franx ${ }^{7}$ (D), Adam Muzzin ${ }^{8}$ (D), David Sobral ${ }^{7,9}$ (D), and Vivienne Wild ${ }^{10}$ \\ ${ }_{1}^{1}$ Max-Planck-Institut für Astronomie, Königstuhl 17, D-69117, Heidelberg, Germany; pofeng@ @mia.de \\ ${ }^{2}$ Sterrenkundig Observatorium, Universiteit Gent, Krijgslaan 281 S9, B-9000 Gent, Belgium \\ ${ }^{3}$ Department of Physics and Astronomy,University of Pittsburgh, 100 Allen Hall, 3941 O'Hara Street, Pittsburgh, PA 15260, USA \\ ${ }_{5}^{4}$ INAF-Osservatorio Astrofisico di Arcetri, Largo Enrico, Fermi 5, I-50125 Firenze, Italy \\ ${ }^{5}$ Space Telescope Science Institute, 3700 San Martin Drive, Baltimore, MD 21218, USA \\ ${ }^{6}$ Department of Astronomy, University of Michigan, 1085 South University Avenue, Ann Arbor, MI 48109-1107, USA \\ ${ }^{7}$ Leiden Observatory, Leiden University, PO Box 9513, 2300 RA Leiden, The Netherlands \\ ${ }^{8}$ Department of Physics and Astronomy, York University, 4700 Keele Street, Toronto, ON M3J 1P3, Canada \\ ${ }^{9}$ Physics Department, Lancaster University, Lancaster LA1 4 YB, UK \\ ${ }^{10}$ School of Physics and Astronomy, University of St Andrews, North Haugh, St Andrews, KY16 9SS, UK \\ Received 2018 June 27; revised 2018 October 9; accepted 2018 October 10; published 2018 November 16
}

\begin{abstract}
We analyze the stellar age indicators $\left(\mathrm{D}_{n} 4000\right.$ and $\left.\mathrm{EW}(\mathrm{H} \delta)\right)$ and sizes of 467 quiescent galaxies with $M_{*} \geqslant 10^{10} M_{\odot}$ at $z \sim 0.7$ drawn from DR2 of the LEGA-C survey. Interpreting index variations in terms of equivalent single stellar population age, we find that the median stellar population is younger for larger galaxies at fixed stellar mass. The effect is significant, yet small; the ages of the larger and smaller subsets differ by only $<500 \mathrm{Myr}$, much less than the age variation among individual galaxies $(\sim 1.5 \mathrm{Gyr})$. At the same time, post-starburst galaxies-those that experienced recent and rapid quenching events - are much smaller than expected based on the global correlation between age and size of normal quiescent galaxies. These coexisting trends unify seemingly contradictory results in the literature; the complex correlations between size and age indicators revealed by our large sample of galaxies with high-quality spectra suggest that there are multiple evolutionary pathways to quiescence. Regardless of the specific physical mechanisms responsible for the cessation of star formation in massive galaxies, the large scatter in $\mathrm{D}_{n} 4000$ and $\mathrm{EW}(\mathrm{H} \delta)$ immediately implies that galaxies follow a large variety of evolutionary pathways. On the one hand, we see evidence for a process that slowly shuts off star formation and transforms star-forming galaxies to quiescent galaxies without necessarily changing their structures. On the other hand, there is likely a mechanism that rapidly quenches galaxies, an event that coincides with dramatic structural changes, producing post-starburst galaxies that can be smaller than their progenitors.
\end{abstract}

Key words: galaxies: evolution - galaxies: formation - galaxies: high-redshift - galaxies: stellar content - galaxies: structure

\section{Introduction}

Large extragalactic surveys have revealed that there are two distinct populations of galaxies: the so-called red sequence, dominated by galaxies with quiescent star formation and old stellar populations, and the blue cloud, containing mainly starforming galaxies (Strateva et al. 2001; Baldry et al. 2004; Bell et al. 2004; Willmer et al. 2006; Franzetti et al. 2007). The structure of the stellar components of these two categories of galaxies shows clear differences. Quiescent galaxies have on average smaller sizes and more concentrated light profiles than star-forming galaxies of the same stellar masses. The average sizes of each population increase by several times from $z \sim 2$ to $z \sim 0$, and the difference in size is in place at all epochs observed (Shen et al. 2003; Trujillo et al. 2007; Williams et al. 2010; van der Wel et al. 2014; Paulino-Afonso et al. 2017). Despite the empirical correlation between galaxy sizes and star formation rates (SFRs) being well established, the physical causality (or lack of; Lilly \& Carollo 2016) remains contentious.

Several mechanisms have been proposed to explain the cessation of star formation in massive galaxies. For field galaxies, one type of mechanism is cutting off the cold gas supply, leading to galaxies naturally running out of fuel for forming new stars. In massive dark matter halos, infalling gas would be shock-heated to high temperature (Dekel \& Birnboim 2006). Additionally, active galactic nuclei (AGNs) or supernovae could inject energy and heat up the gas, further slowing down the cooling process and star formation (Best et al. 2005; Croton et al. 2006; Fabian 2012; Barišić et al. 2017; Terrazas et al. 2017). The star formation may continue for a few Gyr until the cold gas reservoir is used up or heated up.

Another type involves more violent events. Gas-rich galaxy mergers, interactions, and disk instability can efficiently funnel gas into the centers of galaxies, induce intensive star formation, and exhaust the available gas in a short period of time (Barnes \& Hernquist 1991, 1996; Snyder et al. 2011). Gas can also be removed from the galaxies due to the strong outflows induced by starbursts and AGNs (Springel et al. 2005; Kaviraj et al. 2007). Furthermore, the turbulence induced by the violent processes prevents the remaining gas from collapsing and forming stars (Ellison et al. 2018; Smercina et al. 2018).

These two types of processes predict different structural changes during the transition period. The first type of mechanism does not directly involve a change in galaxy structure; therefore, new quiescent galaxies will have sizes similar to their starforming progenitors. The second category involves further star formation activities in the galaxy centers. Dense cores build up during the process, and the structures change. 
The expected sizes of the newly formed quiescent galaxies are thus different in these two scenarios. If the sizes do not change much during the transition from star-forming to quiescent, newly formed quiescent galaxies are expected to be larger than the existing quiescent population. At any epoch, there should be a general correlation between the stellar ages and the sizes of quiescent galaxies such that at fixed stellar mass, larger galaxies are on average younger. This formation process also provides, at least partially, a natural explanation for the smaller average sizes of quiescent galaxies in the early universe. The observed size evolution is a "progenitor bias," in that large quiescent galaxies at the present day were still forming stars at higher redshifts and, thus, are not classified as quiescent galaxies. The population of quiescent galaxies at high redshifts is a smaller biased subset of present-day quiescent galaxies (van Dokkum \& Franx 2001; van der Wel et al. 2009; Carollo et al. 2013; Poggianti et al. 2013). On the other hand, if the quenching process is accompanied by the growth of a dense galaxy core, the effective radii of newly formed quiescent galaxies become smaller than those of their progenitors and can be even smaller than those of existing quiescent galaxies.

The picture is complicated by subsequent merger events. Dry mergers add stars of any ages to the outskirts of quiescent galaxies; therefore, the sizes of quiescent galaxies increase, and the average age of the stellar population is altered. The age-size correlation, if existing at the time quiescent galaxies form, will be gradually washed out by merger events (Shankar et al. 2010). This issue can be mitigated by looking at the highredshift universe, where galaxies are on average much younger, such that fewer merger events would have had happened by the time the galaxies are observed.

Some recent works have attempted to address the age-size correlation up to $z \sim 2$, but the results have so far been inconclusive. Studies splitting the population by size generally report either weak or no significant correlation that larger galaxies are younger (van der Wel et al. 2009; Trujillo et al. 2011; Fagioli et al. 2016; Zanella et al. 2016; Williams et al. 2017). On the contrary, multiple works find that post-starburst (PSB) galaxies, those young quiescent galaxies with SFRs that dropped rapidly in the recent past (Dressler \& Gunn 1983; Balogh et al. 1999; Dressler et al. 1999), are smaller than the average quiescent galaxies (Whitaker et al. 2012; Yano et al. 2016; Almaini et al. 2017). These two statements appear to be supporting different formation and size evolution scenarios of quiescent galaxies.

At the moment, it is not straightforward to combine all of these results and form a coherent picture. Each work studies different galaxy samples in various stellar mass and redshift ranges, and measures the galaxy sizes and ages from imaging and spectroscopic data of different qualities with various methods. In this paper, we present an overview of the sizes and age-sensitive absorption features $\left(\mathrm{D}_{n} 4000\right.$ and $\mathrm{EW}(\mathrm{H} \delta)$ ) of over 400 quiescent galaxies. The sample is drawn from the Large Early Galaxy Astrophysics Census (LEGA-C) survey (van der Wel et al. 2016; Straatman et al. 2018). All galaxies have ultradeep optical spectra that provide precise measurements to infer the ages and recent star formation histories (Chauke et al. 2018; Wu et al. 2018), as well as images from the Hubble Space Telescope (HST) for accurate size measurements.

We will show that the correlations between the size and age indicators are complex and can only be revealed by a large sample of galaxies with high-quality spectra. Our results suggest that galaxies join the red sequence in different ways.

We describe the galaxy sample and the measurement of basic galaxy properties in Section 2. In Section 3, we investigate the correlation between ages and sizes on an individual galaxy basis. In Section 4, we briefly summarize the literature and discuss the implications for the formation of quiescent galaxies. We present our conclusions in Section 5.

\section{Data and Analysis}

This study is based on the first two years of data of the LEGA-C survey. The LEGA-C survey is a four-year survey using the Visible Multi-Object Spectrograph (VIMOS; Le Fèvre et al. 2003) mounted on the $8 \mathrm{~m}$ Very Large Telescope to obtain rest-frame optical spectra of $\sim 3000 K_{s}$-band selected galaxies mainly at $0.6 \leqslant z \leqslant 1.0$. Each galaxy receives $\sim 20 \mathrm{hr}$ of integration at a spectral resolution of $R \sim 3500$. The typical continuum signal-to-noise ratio ( $\mathrm{S} / \mathrm{N})$ is $20 \AA^{-1}$. We refer to van der Wel et al. (2016) for the design of the survey and to Straatman et al. (2018) for details of the observation and data reduction.

\subsection{Stellar Masses, Star Formation Rates, and Sizes of Galaxies}

We derive galaxy stellar masses by fitting the observed multiwavelength spectral energy distributions (SEDs) from the UltraVISTA catalog (Muzzin et al. 2013) using the FAST code (Kriek et al. 2009). The SED templates are from the Bruzual \& Charlot (2003) stellar population synthesis models with exponentially declining SFRs. We adopt a Chabrier (2003) initial mass function (IMF) and the Calzetti et al. (2000) dust extinction law. The SFRs are estimated from the UV and IR luminosities, following the prescription of Whitaker et al. (2012).

We derive the sizes of galaxies using the HST ACS F814W images from the COSMOS program (GO-9822, GO-10092, PI: N. Scoville; Scoville et al. 2007), following the procedure of van der Wel et al. (2012). For each galaxy, we first create a 10" cutout and then use galfit (Peng et al. 2010) to fit a single Sérsic profile to the 2D light profile. The effective radius, total magnitude, Sérsic index, axis ratio, position angle, and background are set as free parameters. Neighboring sources are fit simultaneously. The point-spread function (PSF) is measured from stars in the proximity in terms of the detector position. The size of galaxies, $R_{e}$, is defined as the semimajor axis of the ellipse that contains half of the total flux of the bestfit Sérsic model.

\subsection{Measuring Spectral Indices}

In this paper, we examine two age-sensitive spectral features, the $4000 \AA$ break, $D_{n} 4000$, and the equivalent width of $\mathrm{H} \delta$ absorption, $\mathrm{EW}(\mathrm{H} \delta)$. We use the equivalent width of $\mathrm{H} \beta$ emission to distinguish quiescent from star-forming galaxies.

To separate the ionized gas emission from the stellar continuum, we model the observed spectrum using the Penalized Pixel-Fitting (pPXF) method (Cappellari \& Emsellem 2004; Cappellari 2017). Each galaxy spectrum is fit by a combination of two templates representing the stellar continuum and the gas emission. We model the continuum with high-resolution $(R=10,000)$ theoretical single stellar population (SSP) templates (C. Conroy et al. 2018, in preparation). All emission lines are modeled as a single kinematic component: all have the same 
Table 1

Sample Sizes

\begin{tabular}{lcc}
\hline \hline Quiescence Criteria & $N$ & $N_{\text {PSB }}$ \\
\hline $\mathrm{EW}(\mathrm{H} \beta)>-1 \AA$ & $13(8)$ \\
$\mathrm{sSFR}_{\mathrm{UV}+\mathrm{IR}}<10^{-10} M_{\odot} \mathrm{yr}^{-1}$ & $260(189)$ & $15(13)$ \\
$U V J$ color & $441(372)$ & $35(31)$ \\
\hline
\end{tabular}

Note. The number in the parentheses is the number of spectra covering both $\mathrm{EW}(\mathrm{H} \delta)$ and $\mathrm{D}_{n} 4000$.

velocity and velocity dispersion. The strength of each line is a free parameter. We refer to Bezanson et al. (2018) for the detailed fitting process.

We adopt the definition of $\mathrm{D}_{n} 4000$ in Balogh et al. (1999) and the $\mathrm{H} \delta_{a}$ index in Worthey \& Ottaviani (1997). Both indices are measured from the emission-line-subtracted spectra. The equivalent width of $\mathrm{H} \beta(\mathrm{EW}(\mathrm{H} \beta))$ is the ratio between the bestfit model emission-line flux and the local continuum level, which is defined as the average continuum flux density between $4760 \AA$ and $4960 \AA$ in the rest frame. The typical uncertainties are $\sim 0.025, \sim 0.40 \AA$, and $\sim 0.35 \AA$ for $\mathrm{D}_{n} 4000, \mathrm{EW}(\mathrm{H} \delta)$, and $\mathrm{EW}(\mathrm{H} \beta)$, respectively.

\subsection{Sample Selection}

The first two years of the LEGA-C primary sample consists of 1550 galaxies brighter than $K_{s}=20.7-7.5 \times \log [(1+z) / 1.8]$ and with redshifts $0.6 \leqslant z \leqslant 1.0$ (van der Wel et al. 2016). We exclude spectra (1) with fundamental flaws, (2) that we cannot measure redshifts from, and (3) with a flawed pPXF fit. The remaining 1462 spectra are considered to be useful for scientific purposes and have a flag $f_{\text {use }}=1$ in the LEGA-C DR2 catalog (Straatman et al. 2018).

To measure $\mathrm{EW}(\mathrm{H} \delta)$, we first select 1112 spectra that cover the wavelength range of $\mathrm{EW}(\mathrm{H} \delta)$ and with a median $\mathrm{S} / \mathrm{N} \geqslant 10$ per spectral pixel between rest-frame wavelengths 4000 and $4300 \AA$. The uncertainties on the $\mathrm{EW}(\mathrm{H} \delta)$ of spectra with $\mathrm{S} / \mathrm{N}<10$ are in general $>1 \AA$, too large to be a robust age indicator and would contaminate the selection of PSB galaxies. We then visually inspect $H S T$ and the galfit best-fit model images, excluding 159 galaxies with catastrophic failures in the fitting or highly disturbed morphologies that cannot be well modeled by a single Sérsic profile. From the remaining 953 galaxies, we further exclude 61 galaxies with spectra or broadband photometries contaminated by neighboring objects. In total, we have 892 galaxies with good measurements of spectral indices, sizes, and stellar masses.

Several methods can be used to select quiescent populations, resulting in different levels of contamination and incompleteness (Moresco et al. 2013). In this paper, we analyze three samples of quiescent galaxies using different diagnostics: (1) no or weak $\mathrm{H} \beta$ emission line $(\mathrm{EW}(\mathrm{H} \beta) \geqslant-1 \AA$ ), (2) low specific SFR (sSFR) based on the IR and UV luminosities $\left(\right.$ sSFR $_{\mathrm{UV}+\mathrm{IR}}<10^{-10} M_{\odot} \mathrm{yr}^{-1}$ ), and (3) the rest-frame $U-V$ and $V-J$ colors, the demarcation of Muzzin et al. (2013). Table 1 lists the sample sizes with the three definitions of quiescence. The $\operatorname{EW}(\mathrm{H} \beta)$ sample contains fewer galaxies because of an extra constraint on the spectral coverage; the spectra have to cover rest frame $\sim 5000 \AA$ in order to measure the $\mathrm{EW}(\mathrm{H} \beta)$, which is not required for the $\mathrm{UV}+\mathrm{IR}$ sSFR and the $U V J$ color selections. The number in the parentheses is the number of spectra that also cover the $\mathrm{D}_{n} 4000$ region. When $\mathrm{D}_{n} 4000$ is required for the analysis, we use this subset of data.
These three classifications in general agree with each other well (Figure 1). In each sample, $\sim 90 \%$ of the galaxies are also defined as quiescent by the other two criteria. We estimate the "incompleteness" by calculating the number of star-forming galaxies that would be defined as quiescent galaxies by either of the other two criteria. The number of these potential missing objects is $<15 \%$ of the number of quiescent galaxies.

We adopt the $\mathrm{H} \beta$ emission line as our fiducial criteria of quiescence, as the Balmer emission lines trace star formation in a shorter timescale $(\sim 10 \mathrm{Myr})$ compared to the other two indicators ( $\gtrsim 100 \mathrm{Myr}$ ). We note that we do not adopt the [O II] $\lambda 3727,3729$ doublets as a star formation indicator. It has been shown that roughly half of the red galaxies and PSB galaxies have moderate [O II] emission while lacking Balmer emission. The excess [O II] is likely due to AGNs or LINERs rather than star formation activities. Requiring no or weak [O II] emission results in a highly incomplete and potentially biased sample of quiescent galaxies (Yan et al. 2006; Lemaux et al. 2010; Wu et al. 2014; Lemaux et al. 2017).

We repeat the analysis using the UV+IR sSFR and $U V J$ color quiescent samples and show the complementary results in the Appendix. The conclusions in this paper are not affected by the definition of quiescence. Figure 2 shows the redshift and stellar mass distributions of the $\operatorname{EW}(\mathrm{H} \beta)$ quiescent sample. We also plot the distributions of $U V J$ quiescent galaxies for a comparison. The $\operatorname{EW}(\mathrm{H} \beta)$ quiescent galaxies are at lower redshifts because the $\mathrm{H} \beta$ line is generally outside our spectral coverage at $z \gtrsim 0.8$; we are not able to use the $\mathrm{H} \beta$ selection for those galaxies.

\subsection{Definition of Post-starburst Galaxies}

PSB galaxies are galaxies whose star formation was shut off rapidly in the last $\sim 1$ Gyr. One of the prominent spectral features of PSB galaxies is their strong Balmer absorptions, indicating a dominating population of A- to early F-type stars (Dressler \& Gunn 1983; Balogh et al. 1999; Dressler et al. 1999).

In order to select PSB galaxies, several methods have been proposed for different types of data (e.g., Wild et al. 2009; Kriek et al. 2010; Muzzin et al. 2012). Our spectra allow us to measure the $\mathrm{H} \delta$ absorption strength accurately. Therefore, we use a traditional definition and define PSB galaxies as quiescent galaxies with strong $\mathrm{H} \delta$ absorption. The boundary of "strong" $\mathrm{H} \delta$ is often drawn at $\mathrm{EW}(\mathrm{H} \delta)=3$ to $\sim 5 \AA$. In this paper, we require PSB galaxies to have $\mathrm{EW}(\mathrm{H} \delta)>4 \AA$. The numbers of PSB galaxies with each definition of quiescence are listed in Table 1 .

\subsection{Mass-Size Relation}

Figure 3 shows the mass-size relations of quiescent galaxies. We fit a linear relation between the median sizes in each 0.1 dex mass bin and the mass in log-log space:

$$
\log \left(R_{e} / \mathrm{kpc}\right)_{\mathrm{med}}=a \times\left[\log \left(M_{*} / M_{\odot}\right)-11\right]+b \text {. }
$$

We fit both for the whole sample and the $\mathrm{D}_{n} 4000$ subset, and estimate the uncertainties from bootstrap resampling. The two best-fit parameters $(a, b)$ are $\left(0.51_{-0.06}^{+0.06}, 0.63_{-0.01}^{+0.01}\right)$ and $\left(0.55_{-0.03}^{+0.04}, 0.63_{-0.01}^{+0.01}\right)$, respectively. The two best-fit relations agree with each other within $0.04 \mathrm{dex}$ in $R_{e}$ at fixed mass. 

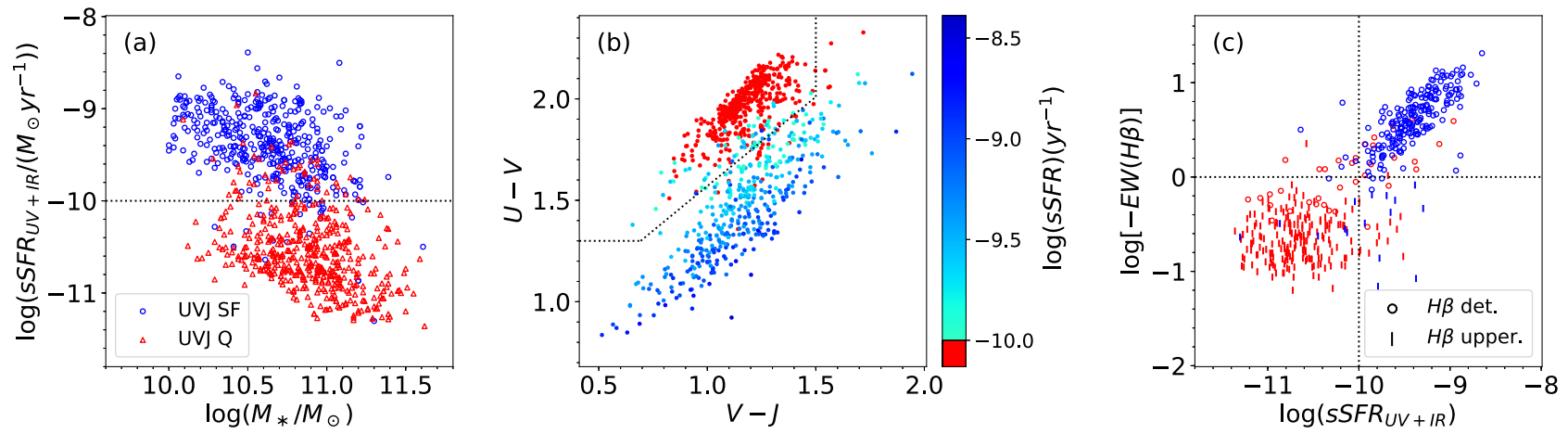

Figure 1. Comparison of three different methods separating star-forming and quiescent galaxies: the equivalent width of the $\mathrm{H} \beta$ emission line, sSFR derived from UV and IR fluxes, and the $U V J$ two-color scheme. (a) The sSFR as a function of stellar mass. We adopt a fiducial sSFR limit of $10^{-10} M_{\odot} \mathrm{yr}^{-1}$ separating star-forming and quiescent galaxies (the dotted horizontal line). Blue circles and red triangles are star-forming and quiescent galaxies based on the $U V J$ two-color scheme. (b) The $U-V$ vs. $V-J$ two-color diagram. Galaxies are color-coded according to the sSFR. The dotted line labels the demarcation separating star-forming from quiescent galaxies of Muzzin et al. (2013). Galaxies at the top-left corner are classified as quiescent galaxies based on the two-color scheme. (c) EW(H $\beta$ ) as a function of sSFR. The circles are galaxies with detected $\mathrm{H} \beta$ emission. The short vertical lines are $2 \sigma$ upper limits of the non-detections. The vertical and horizontal dotted lines are the sSFR and $\operatorname{EW}(\mathrm{H} \beta)$ demarcations we adopted in the paper. Blue and red data points are star-forming and quiescent galaxies based on the $U V J$ two-color scheme. Panel (c) shows that the three different methods, in general, agree with each other well.
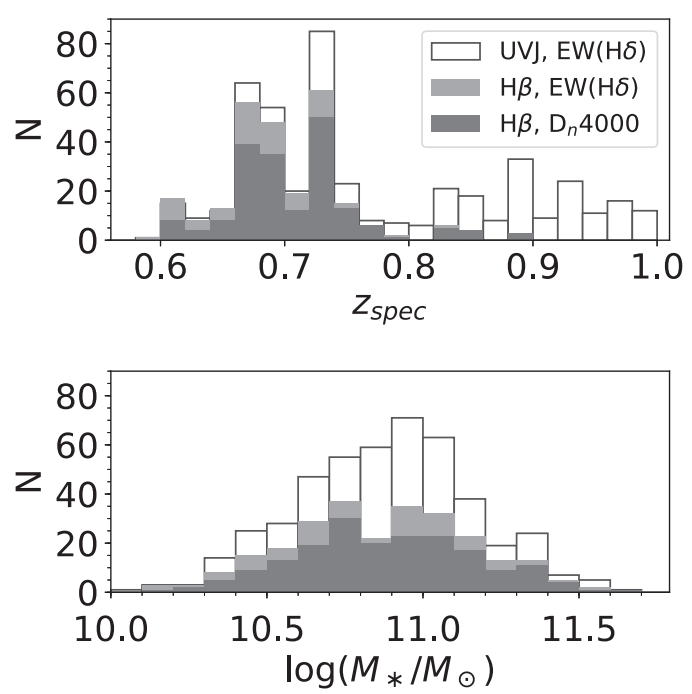

Figure 2. Distributions of redshifts and stellar masses of quiescent galaxies. The light and dark gray histograms are the distributions of galaxies with only $\mathrm{EW}(\mathrm{H} \delta)$ and both $\mathrm{EW}(\mathrm{H} \delta)$ and $\mathrm{D}_{n} 4000$ measurements, respectively. The distributions of the $U V J$ quiescent sample is also presented for a comparison. The $\operatorname{EW}(\mathrm{H} \beta)$ quiescent galaxies have $z \lesssim 0.8$ because the $\mathrm{H} \beta$ line shifts outside of our spectral coverage at $z \gtrsim 0.8$. We are not able to use $\mathrm{H} \beta$ to select quiescent galaxies.

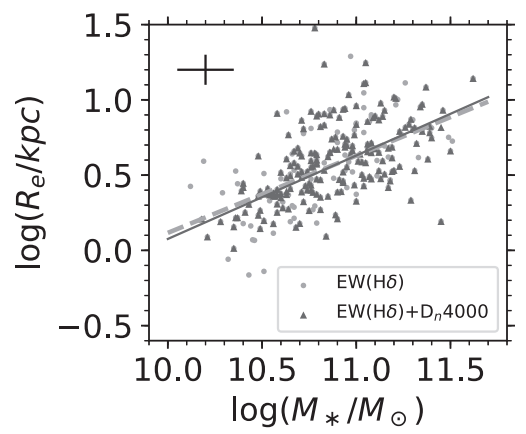

Figure 3. Sizes and stellar masses of quiescent galaxies. Dark gray triangles are galaxies with spectra covering both $\mathrm{D}_{n} 4000$ and $\mathrm{EW}(\mathrm{H} \delta)$. Light gray circles are galaxies for which only $\mathrm{EW}(\mathrm{H} \delta)$ is measurable. The light gray dashed line and the dark gray solid line are the best-fit mass-size relation for all data and black points, respectively. The two best-fit relations are consistent with each other within 0.04 dex.

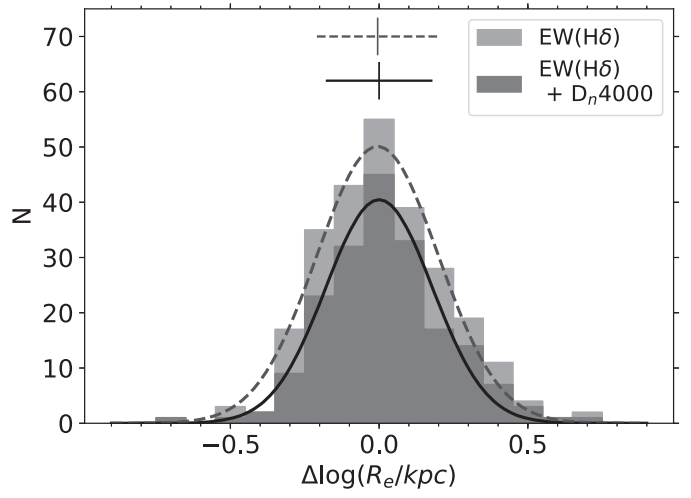

Figure 4. Distributions of $\Delta \log \left(R_{e}\right)$, the sizes relative to the median sizes at fixed stellar mass. The light gray histogram is the distribution of the whole quiescent sample. The dark gray histogram is the subset of galaxies that also have $\mathrm{D}_{n} 4000$ measurements. The dashed and solid curves are the best-fit Gaussian models to the two distributions. The distributions are well represented by Gaussian distributions centered at $\sim 0$ and with standard deviations of $\sim 0.2$ dex as labeled at the top of the panel.

In this paper, we use the sizes relative to the median sizes at fixed masses as the metric for galaxy sizes:

$$
\Delta \log \left(R_{e}\right)=\log \left(R_{e}\right)-\log \left(R_{e}\right)_{\mathrm{med}},
$$

where $\log \left(R_{e}\right)_{\text {med }}$ is the best-fit median size at the given masses according to Equation (1) and the best-fit parameters of each sample, respectively. The distributions of $\Delta \log \left(R_{e}\right)$ can be modeled as a Gaussian distribution centered at $\sim 0$, with a standard deviation of $\sim 0.2$ dex (Figure 4 ).

We note that our best-fit slope is different from the slope derived from the CANDLES survey (0.71; van der Wel et al. 2014). The difference can be the result of different sample selections and fitting methods. We checked that the $\mathrm{S} / \mathrm{N}$ cut does not bias the size. The $\Delta \log \left(R_{e}\right)$ distribution of low-S/N galaxies with good mass and size measurements can be well modeled by a Gaussian centered at $\sim 0$ with a standard deviation of $\sim 0.2$ dex, similar to Figure 4 . We also repeat the fitting using samples without the $\mathrm{S} / \mathrm{N}$ cut and find that the slopes change by $<0.03$, and the best-fit $R_{e}$ at fixed masses change by $<0.04$ dex. 

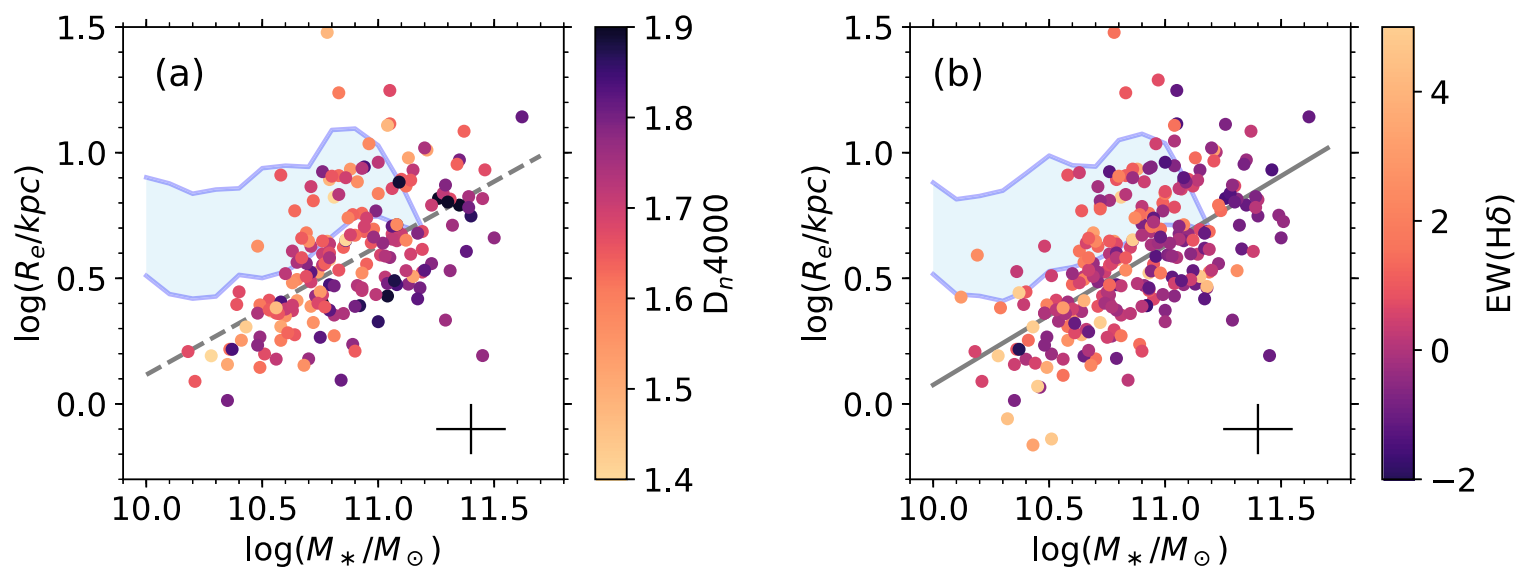

Figure 5. Masses and sizes of quiescent galaxies, color-coded by $\mathrm{D}_{n} 4000$ and $\mathrm{EW}(\mathrm{H} \delta)$. At any fixed mass and size, galaxies can have a wide range of $\mathrm{D}_{n} 4000$ and $\mathrm{EW}(\mathrm{H} \delta)$, indicating a large variation in stellar ages among galaxies of similar masses and sizes. There is a tentative trend such that more massive galaxies and smaller galaxies have larger $\mathrm{D}_{n} 4000$. The $\mathrm{EW}(\mathrm{H} \delta)$ has a weak dependence on mass, where more massive galaxies have smaller $\mathrm{EW}(\mathrm{H} \delta)$. The dependence on the size is not clear. The light blue shaded areas are the 16th and 84th percentiles of the sizes of star-forming galaxies at fixed masses. The dashed and solid lines are the best-fit mass-size relations of quiescent galaxies. The cross in the bottom-right corner represents the uncertainties in stellar mass $(0.15$ dex $)$ and sizes $(0.10$ dex $)$.

\section{Correlations between Sizes and Age-sensitive Indicators}

We examine the size dependences of two age-sensitive spectral features, $\mathrm{D}_{n} 4000$ and $\mathrm{EW}(\mathrm{H} \delta)$. For passive galaxies, $\mathrm{D}_{n} 4000$ increases monotonically with the stellar age. On the other hand, $\mathrm{EW}(\mathrm{H} \delta)$ is sensitive to recent star formation such that galaxies with rapidly declining SFRs will have elevated $\mathrm{EW}(\mathrm{H} \delta)$ in the first few hundred Myr after star formation stops, and then $\mathrm{EW}(\mathrm{H} \delta)$ gradually decreases afterward. These two indices together work as proxies for stellar ages and recent star formation histories (Kauffmann et al. 2003; Wu et al. 2018).

\subsection{The Dependence of $D_{n} 4000$ and $E W(H \delta)$ on Galaxy Size}

Figure 5 shows the stellar masses and sizes of quiescent galaxies, color-coded by $\mathrm{D}_{n} 4000$ and $\mathrm{EW}(\mathrm{H} \delta)$. For comparison, we also plot the size distribution of star-forming galaxies (16th and 84th percentiles) as a function of stellar masses. First of all, at a given mass and size, galaxies can have very different $\mathrm{D}_{n} 4000$ and $\mathrm{EW}(\mathrm{H} \delta)$. The typical uncertainty of our $\mathrm{D}_{n} 4000$ and $\mathrm{EW}(\mathrm{H} \delta)$ measurement is $\sim 0.025$ and $\sim 0.4 \AA$, much smaller than the range of the distribution. The large color variations in Figure 5 are not due to measurement uncertainties but suggest large individual variations in ages at fixed stellar mass and size.

Despite the large individual variation, a weak trend can be identified in Figure 5(a). Galaxies with a large $\mathrm{D}_{n} 4000$, those with dark colors, tend to be more massive and smaller than the average. The upper half of the mass-size relation is occupied mainly by points with lighter color, those galaxies with small $\mathrm{D}_{n} 4000$. As for $\mathrm{EW}(\mathrm{H} \delta)$ in Figure 5(b), a weak mass dependence is present such that high-mass galaxies have on average smaller $\mathrm{EW}(\mathrm{H} \delta)$. On the other hand, by visual inspection, there is no clear size dependence.

We apply the Locally Weighted Regression (LOESS) method to derive the mean trends of $\mathrm{D}_{n} 4000$ and $\mathrm{EW}(\mathrm{H} \delta)$ on the mass-size plane (Figure 6). We use the CAP_LOESS_2D routine of Cappellari et al. (2013), which implements the multivariate LOESS algorithm of Cleveland \& Devlin (1988). We adopt a regularization factor $f=0.5$ and a linear local approximation.

The LOESS-smoothed maps confirm the visual inspection of Figure 5. First, more massive galaxies have on average larger
$\mathrm{D}_{n} 4000$ and smaller $\mathrm{EW}(\mathrm{H} \delta)$, suggesting that massive galaxies form their stars earlier (see Haines et al. 2017; Siudek et al. 2017; Wu et al. 2018 for results at similar redshifts). At fixed stellar mass, larger galaxies have smaller $\mathrm{D}_{n} 4000$ (see Zahid \& Geller 2017 for results at lower redshifts). On the other hand, the behavior of $\mathrm{EW}(\mathrm{H} \delta)$ is more complex. For massive galaxies $\left(M_{\odot} \gtrsim 10^{11} M_{\odot}\right)$, the LOESS-smoothed map does not show a clear trend in $\mathrm{EW}(\mathrm{H} \delta)$ as a function of size. For the bulk of lower mass galaxies, larger galaxies have slightly larger $\mathrm{EW}(\mathrm{H} \delta)$. However, we also find that the smallest bins have large $\mathrm{EW}(\mathrm{H} \delta$ ) (see also Figures 16 and 17 in the Appendix). This reversal is driven by a few small galaxies with very strong $\mathrm{H} \delta$ absorption (Figure 5). Figure 7(a) shows an example. Comparing to galaxies of similar masses, the Balmer absorption is clearly stronger than that of galaxies of similar size (Figure 7(b)) or much larger (Figure 7(c)). We discuss the correlation between the spectral indices and the size of galaxies in further detail in the following sections.

\subsection{The Correlation between Stellar Ages and Sizes of Quiescent Galaxies}

Figure 8 shows the relations between $\Delta \log \left(R_{e}\right)$, the size relative to the median sizes at fixed mass (Section 2.5), and $\mathrm{D}_{n} 4000$ and $\mathrm{EW}(\mathrm{H} \delta)$ for the whole sample and in two stellar mass bins. As in Figure 5, at fixed $\Delta \log R_{e}$, quiescent galaxies can have very different $\mathrm{D}_{n} 4000$ and $\mathrm{EW}(\mathrm{H} \delta)$. The light gray shaded areas in Figure 8, the 16th and the 84th percentiles of the $\mathrm{D}_{n} 4000$ and $\mathrm{EW}(\mathrm{H} \delta)$ distributions, cover $\mathrm{D}_{n} 4000 \simeq$ 1.6-1.8 and $\mathrm{EW}(\mathrm{H} \delta) \simeq-1 \AA-2 \AA$, respectively. For a simple stellar population SSP with solar metallicity (Bruzual \& Charlot 2003), these ranges correspond to an age range of $\sim 1.5$ Gyr. The running medians (black lines) show that $\mathrm{D}_{n} 4000$ progressively decreases as $\Delta \log \left(R_{e}\right)$ increases. The overall size dependence in $\mathrm{EW}(\mathrm{H} \delta)$ is less significant. For the low-mass bin, larger galaxies have on average slightly larger $\mathrm{EW}(\mathrm{H} \delta)$, except for the most compact galaxies. There is no detectable size dependence for the high-mass bin.

We show the median indices of galaxies in four size bins on the $\mathrm{D}_{n} 4000-\mathrm{EW}(\mathrm{H} \delta)$ plane (Figure 9). The size bins are divided at $\Delta \log \left(R_{e}\right)=-0.2,0$, and 0.2 . The middle two size bins contain the majority of galaxies as $0.2 \mathrm{dex}$ is the $1 \sigma$ of the distribution of $\Delta \log \left(R_{e}\right)$ (Section 2.5). The largest and the 

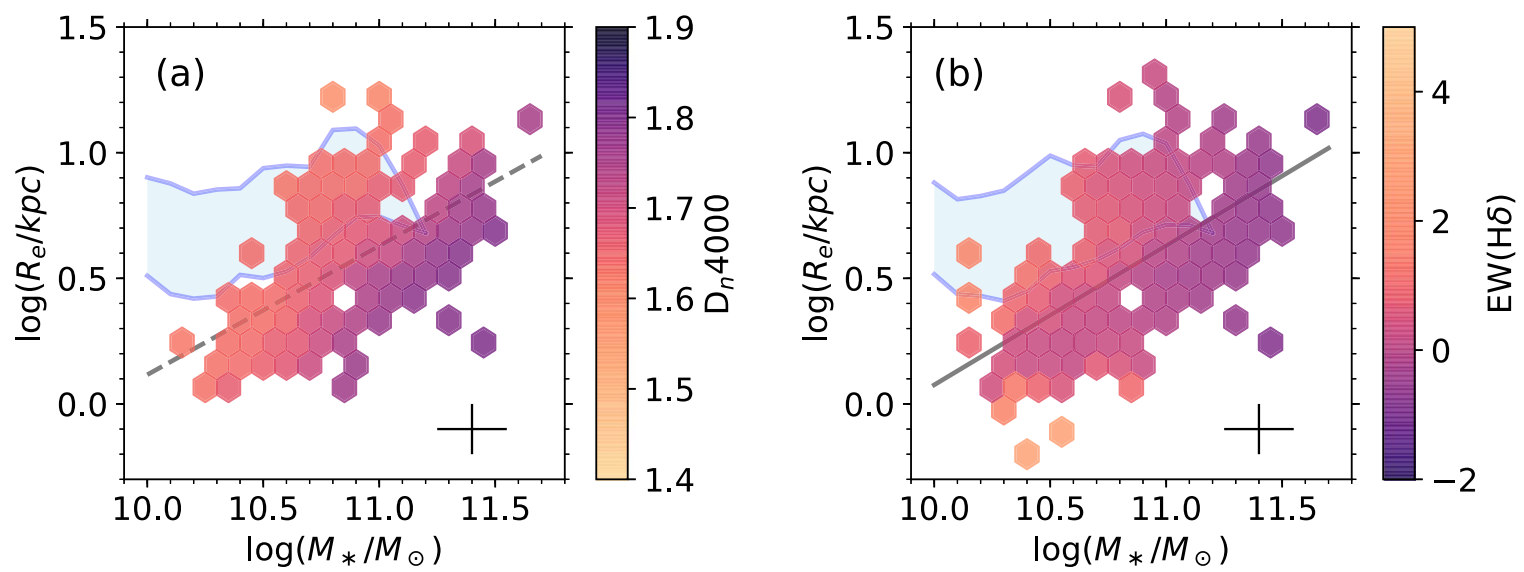

Figure 6. $\mathrm{D}_{n} 4000$ and $\mathrm{EW}(\mathrm{H} \delta)$ averaging over nearby data points on the mass-size plane using the LOESS method (see the text). The smoothed maps reveal the underlying size dependence. Larger galaxies have on average smaller $\mathrm{D}_{n} 4000$ and larger $\mathrm{EW}(\mathrm{H} \delta)$. However, the most compact galaxies also have large $\mathrm{EW}(\mathrm{H} \delta)$, which is opposite to the general trend. The light blue shaded areas are the 16th and 84th percentiles of the sizes of star-forming galaxies at fixed masses. The dashed and solid lines are the best-fit mass-size relations of quiescent galaxies. Complementary results using other definitions of quiescence are shown in Figures 16 and 17 in the Appendix.

smallest bins, on the other hand, contain galaxies with sizes significantly larger or smaller than the average population. We also plot the evolutionary model track of the solar-metallicity SSP for reference. The trend in $\mathrm{D}_{n} 4000$ and $\mathrm{EW}(\mathrm{H} \delta$ ) combined suggests that except for the smallest bin, there is a weak size dependence on stellar ages that larger galaxies are on average younger, but the difference in age is small, $<500 \mathrm{Myr}$. Galaxies in the smallest size bin are a mix of old galaxies and PSB galaxies (See Section 3.3).

We also investigate how the sizes of galaxies change as a function of $\mathrm{D}_{n} 4000$ or $\mathrm{EW}\left(\mathrm{H} \delta\right.$ ) (Figure 10). $\Delta \log \left(R_{e}\right)$ increases toward smaller $\mathrm{D}_{n} 4000$ and larger $\mathrm{EW}(\mathrm{H} \delta)$ at $\mathrm{D}_{n} 4000 \gtrsim 1.6$ and $\mathrm{EW}(\mathrm{H} \delta) \lesssim 2 \AA$, the regimes where the quiescent galaxies are the dominating population (Figure 8 and Kauffmann et al. 2003; Haines et al. 2017; Wu et al. 2018). Within these ranges of indices, the Spearman's rank correlation coefficients $\rho$ and their $p$-values between $\Delta \log \left(R_{e}\right)$ and the indices are $\rho=-0.37$, $p$-value $=3 \times 10^{-6}$ for $\mathrm{D}_{n} 4000$ and $\rho=0.19, \quad p$-value $=$ $4 \times 10^{-3}$ for $\mathrm{EW}(\mathrm{H} \delta)$. We fit linear relations to the median $\Delta \log \left(R_{e}\right)$ and the indices for quiescent galaxies with $\mathrm{D}_{n} 4000 \geqslant$ 1.6 and $\mathrm{EW}(\mathrm{H} \delta) \leqslant 2 \AA$ in Figures $10(\mathrm{a})$ and (d) as

$$
\begin{aligned}
\Delta \log \left(R_{e}\right)= & {\left[(-0.68 \pm 0.08) \times \mathrm{D}_{n} 4000\right] } \\
& +(1.17 \pm 0.14) \\
\Delta \log \left(R_{e}\right)= & {[(0.065 \pm 0.005) \times \mathrm{EW}(\mathrm{H} \delta)] } \\
& +(-0.001 \pm 0.005) .
\end{aligned}
$$

These results suggest a trend that quiescent galaxies with younger stellar populations are on average larger, consistent with Figures 8 and 9.

However, the median sizes become smaller at $\mathrm{EW}(\mathrm{H} \delta)>2 \AA$, reaching $\Delta \log \left(R_{e}\right) \simeq-0.2$ at $\mathrm{EW}(\mathrm{H} \delta) \simeq 4 \AA$ (Figures $10(\mathrm{~d}$ ) and (f)). We will discuss the sizes of these PSB galaxies in the next section.

\subsection{The Size of Post-starburst Galaxies}

Figure 11 shows PSB galaxies on the mass-size plane. The majority (10/13) of PSB galaxies are located at the bottom half of the mass-size plane, and $\sim 40 \%(5 / 13)$ are below the $1 \sigma$ of the size distribution (see Figure 21 in the Appendix for complementary results). The PSB galaxies have median $\mathrm{D}_{n} 4000 \simeq 1.45$ and median $\mathrm{EW}(\mathrm{H} \delta) \simeq 5.2 \AA$. Casting the indices in terms of the ages of an SSP, they are among the youngest quiescent galaxies but they are not larger than the average quiescent population.

The whole PSB population has a median size of $\Delta \log \left(R_{e}\right)=$ $-0.07 \pm 0.07$. By extrapolating Equation (3), the expected sizes of galaxies with $\mathrm{EW}(\mathrm{H} \delta) \simeq 5 \AA$ will be $\Delta \log \left(R_{e}\right) \simeq 0.3$, which is much larger than PSB galaxies but comparable to starforming galaxies (see Figure 10(d)). Even comparing to the sizes of typical young quiescent galaxies $\left(\Delta \log \left(R_{e}\right) \simeq 0.13\right.$ at $\mathrm{EW}(\mathrm{H} \delta) \simeq 2 \AA$ ), $\mathrm{PSB}$ galaxies are still significantly smaller; they do not follow the general correlation between the age and the size characterized by the majority of galaxies.

\section{Discussion}

The results in Section 3 can be summarized as follows: at fixed mass, (1) larger quiescent galaxies have on average younger stellar ages, and (2) PSB galaxies are much smaller than expected based on their young stellar populations. The correlation among the sizes, the ages, and the recent star formation histories puts constraints on the formation and evolution processes of quiescent galaxies. Several recent studies address this question but report seemingly contradictory results. In this section, we show that our high-quality data and the larger sample size present a complete picture. We then discuss the implication for the formation of quiescent galaxies.

\subsection{Reconciling Tensions in the Literature}

Several studies have investigated the age-size correlation of quiescent galaxies. Fagioli et al. (2016) and Williams et al. (2017) split their samples into two size bins and derive galaxy ages from co-added spectra in each size bin. They find that at $M_{*}<10^{11} M_{\odot}$, smaller galaxies are slightly older, but the age difference between galaxies in the two size bins is at largest a few hundred Myr at $0.2<z<1.2$. For galaxies with $M_{*}>$ $10^{11} M_{\odot}$, there appears to be no clear trend. Belli et al. (2015) shows that at $1<z<1.5$, quiescent galaxies older than $1.25 \mathrm{Gyr}$ are smaller than the average at fixed stellar masses, whereas Trujillo et al. (2011) and Zanella et al. (2016) find no significant size difference between old and young quiescent galaxies at $z<2$. 


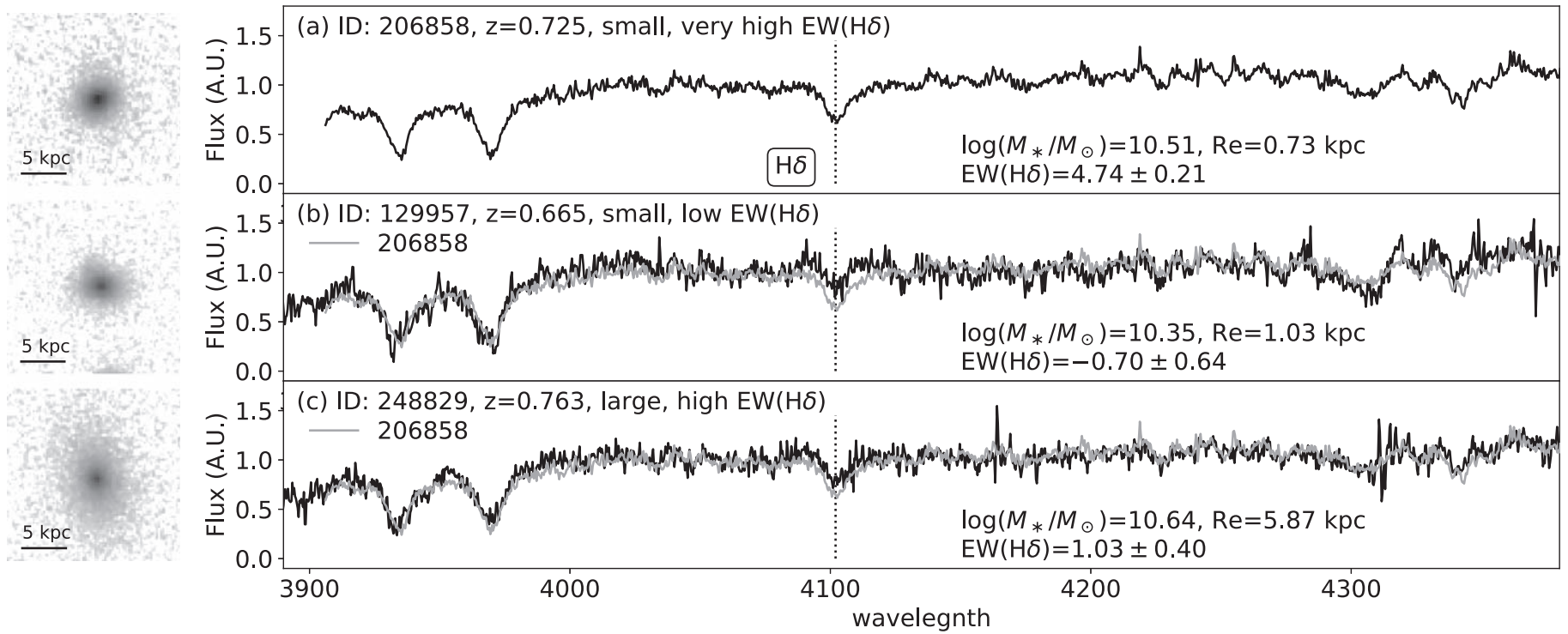

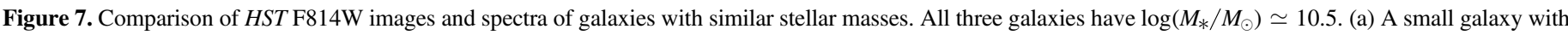

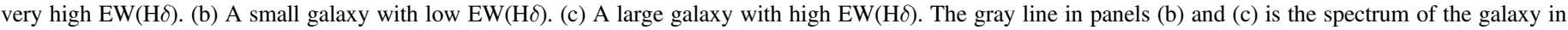

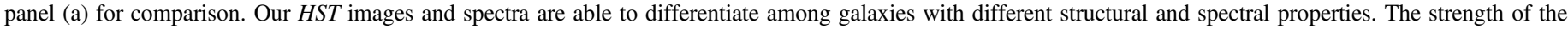
$\mathrm{EW}(\mathrm{H} \delta)$ absorption of the galaxy in panel (a), despite its small size, is clearly stronger than that of the other two galaxies.

On the other hand, Keating et al. (2015) find that compact massive galaxies $\left(r<2 \mathrm{kpc}\right.$ and $\left.M_{*}>10^{11} M_{\odot}\right)$ at $z \sim 1$ are younger than a control sample of galaxies of similar masses. A few studies on high- $z$ PSB galaxies also find that these galaxies are among the smallest quiescent galaxies at fixed stellar masses (Whitaker et al. 2012; Yano et al. 2016; Almaini et al. 2017). These studies based on either the most compact galaxies or PSB galaxies conclude that quiescent galaxies were compact when they formed.

There is apparent tension between these statements. Our results in Section 3 present a complete picture that reconciles these seemingly contradictory observations. First of all, for the three largest size bins, the median $\mathrm{D}_{n} 4000$ and $\mathrm{EW}(\mathrm{H} \delta)$ differ by $\sim 0.06$ and $\sim 0.5 \AA$. When casting these age indicators in terms of SSP ages, the ages differ by only $<500 \mathrm{Myr}$ (Figure 9). The difference in age is in broad agreement with previous studies derived from stacking spectra of large and small galaxies (Trujillo et al. 2011; Fagioli et al. 2016; Williams et al. 2017). At the same time, we also find that PSB galaxies are much smaller than expected based on the global correlation between age and size.

The apparent contradiction in the literature is mainly due to sample selection and data binning that present only a partial view. First of all, studies focusing on special objects, either compact galaxies or PSB galaxies, pick up outliers that do not follow the average age-size correlation (Figure 10). Second, the individual age variation is large compared to the average trend (Figure 8). The correlation between the age indicators and the sizes may not be detectable using a small sample or low$\mathrm{S} / \mathrm{N}$ data. The stacking analysis can improve the measurements and recover the average trend when a large sample is available, but wash out the variations among individual galaxies. PSB galaxies represent only $\sim 5 \%$ of the quiescent population in our sample (13 out of 260 in our sample; see also Vergani et al. 2008; Yan et al. 2009; Muzzin et al. 2012; Wu et al. 2014; Wild et al. 2016 for studies at $z \sim 1$ ). The stacking analysis cannot identify the peculiar behaviors of these rare special objects. Only with LEGA-C's unique combination of sample size and data quality can we reveal the complex correlations between galaxy sizes and age indicators.

\subsection{Multiple Ways to Quiescence}

The different sizes of PSB galaxies and the rest of young quiescent galaxies suggest that these populations have different star formation histories. By definition, the star formation activities in PSB galaxies have been shut off rapidly within a short timescale of a few hundred Myr (Le Borgne et al. 2006; Wild et al. 2009; Snyder et al. 2011). On the contrary, other young quiescent galaxies did not necessarily experience the same rapid quenching processes. The correlation between the size and the timescale that star formation shuts off corresponds to different types of evolutionary scenarios.

\subsubsection{Slow Process, Large Young Quiescent Galaxies}

The first type of mechanisms to stop star formation is cutting off the supply of cold gas; therefore, galaxies naturally run out of fuel to form new stars. In massive dark matter halos, cold gas can be shock-heated to high temperature while falling into the halos (Kereš et al. 2005; Dekel et al. 2009). Furthermore, AGNs can inject energy and keep the halo gas hot (Best et al. 2005, 2014; Croton et al. 2006; Sijacki et al. 2007; Yesuf et al. 2014; Terrazas et al. 2016; Barišić et al. 2017). The SFRs thus gradually drop as the cold gas reservoir is depleted. This process does not directly require a change in structure when a galaxy transforms from star-forming to quiescent.

At fixed stellar mass, the size distributions of star-forming and quiescent galaxies overlap with each other in such a way that the smaller half of star-forming galaxies have similar sizes to the larger half of quiescent galaxies (van der Wel et al. 2014, also Figure 11). The similarity in stellar masses and sizes makes small star-forming galaxies candidate immediate progenitors of quiescent galaxies. As the SFR declines, a relatively small star-forming galaxy naturally becomes a relatively large quiescent galaxy with young stellar 


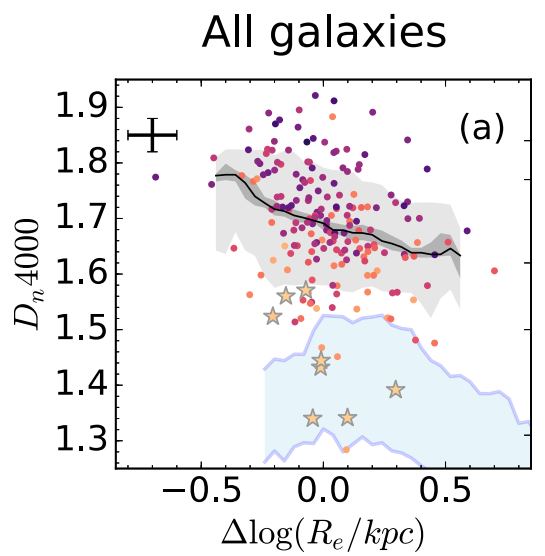

$$
\log \left(M_{*} / M_{\odot}\right) \geq 11
$$

$\log \left(M_{*} / M_{\odot}\right)<11$
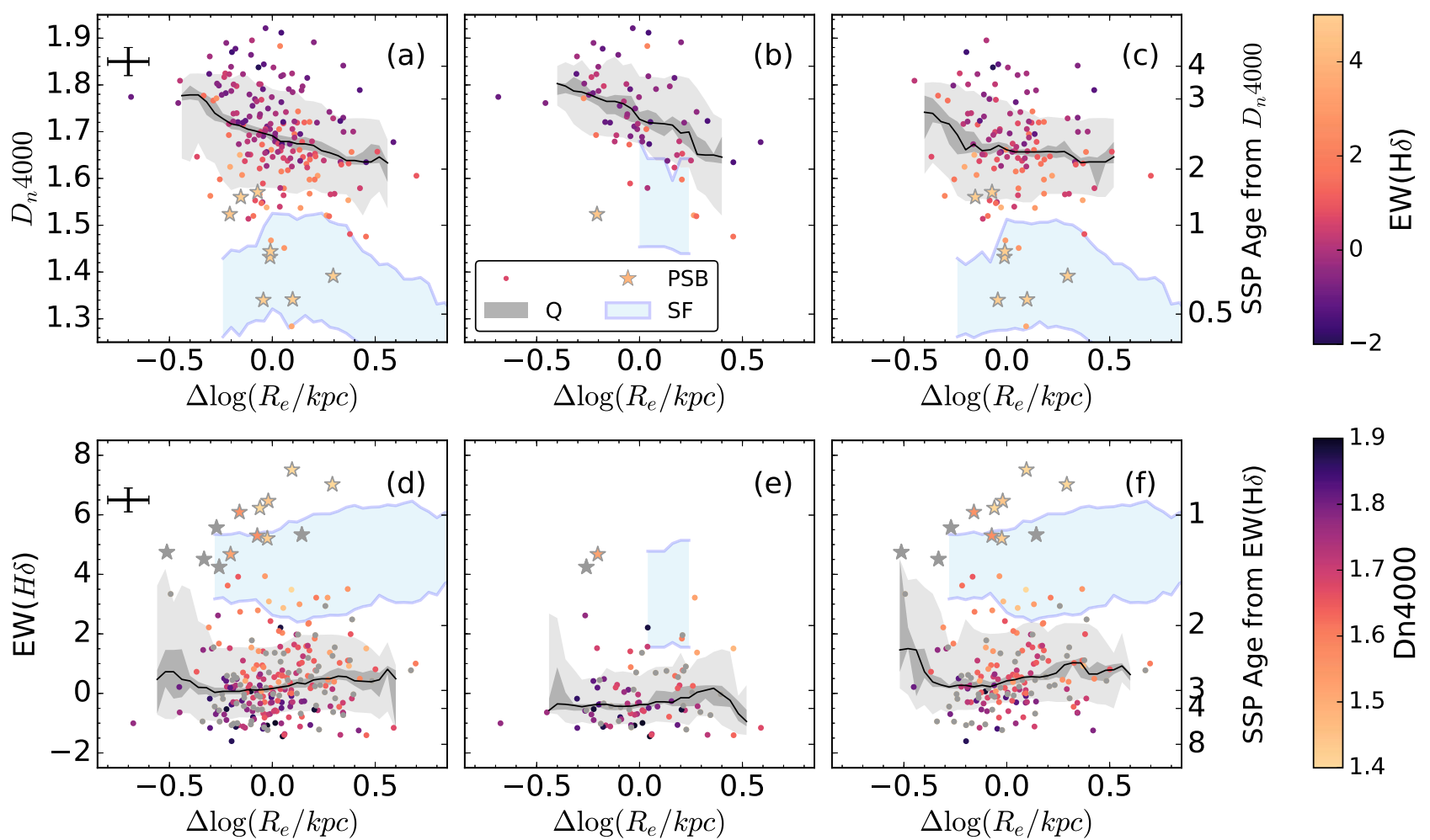

Figure 8. Correlation between the size and the absorption line indices $\mathrm{D}_{n} 4000$ and $\mathrm{EW}(\mathrm{H} \delta$ ) of all (left), high-mass (center), and low-mass (right) quiescent galaxies. PSB galaxies are labeled as stars. In panels (a)-(c), the colors show the $\mathrm{EW}(\mathrm{H} \delta)$ value. In panels (d)-(f), the colors show $\mathrm{D}_{n} 4000$. Gray points are galaxies without $\mathrm{D}_{n} 4000$ measurements. The solid black lines show the running median of $\mathrm{D}_{n} 4000$ and $\mathrm{EW}(\mathrm{H} \delta)$ as a function of $\Delta \log \left(R_{e}\right)$, the sizes relative to the median sizes at fixed masses. The light gray areas indicate the 16th and 84th percentiles of the distributions at fixed $\Delta \log \left(R_{e}\right)$. The dark gray areas are the uncertainties of the medians, estimated from 1000 bootstrap samples. The light blue area shows the 16th and 84th percentiles of the indices of star-forming galaxies. The $y$-axis on the right-hand side labels the corresponding ages of a solar-metallicity SSP of each index. Star-forming galaxies have on average smaller $\mathrm{D}_{n} 4000$, larger $\mathrm{EW}(\mathrm{H} \delta)$, and larger sizes than quiescent galaxies. We only show intervals with more than five galaxies within a 0.2 dex size bin. Overall, the $\mathrm{D}_{n} 4000$ of quiescent galaxies decreases as $\Delta \log \left(R_{e}\right)$ increases, indicating that larger galaxies are on average younger. There is no clear size dependence on $\operatorname{EW}(\mathrm{H} \delta)$. At fixed $\Delta \log \left(R_{e}\right)$, the distributions of $\mathrm{D}_{n} 4000$ and $\mathrm{EW}(\mathrm{H} \delta)$ correspond to an age range of $\sim 1.5 \mathrm{Gyr}$ assuming a solar-metallicity SSP.

populations. The average size of the quiescent population also increases.

Carollo et al. (2013) calculated the average size growth of the quiescent population at $z<1$ under the assumption that at any time, newly formed quiescent galaxies have the same size distributions as the star-forming galaxies at the same epoch. They are able to reproduce the size evolution of massive $\left(M_{*}>10^{11} M_{\odot}\right)$ quiescent galaxies but overpredict the sizes of lower mass quiescent galaxies. If small star-forming galaxies are progenitors of newly formed quiescent galaxies, this discrepancy can be mitigated.

In the local universe, where the total cold gas mass is measurable, the gas content in star-forming galaxies strongly correlates with the size of stellar disks, such that at fixed stellar mass, star-forming galaxies with more compact disks possess less gas (Wu 2018). This dependence of gas content on disk sizes is likely in place up to $z \sim 3$ based on an inversion of a star formation law (Popping et al. 2015). Once there is no more cold gas supply, smaller star-forming galaxies would use up the fuel quicker.

Moreover, from a dynamical point of view, more compact, high surface density galaxies are expected to be less stable against both global and local growth of instability, and thus more efficient in turning gas into stars (Dalcanton et al. 1997; Mihos et al. 1997). This size dependence of star formation efficiency is also required to explain the size dependence of gas-phase metallicity (Wu et al. 2015). The instability may also trigger the gas flows toward the centers of galaxies, which accelerate the growth of black holes (Bournaud et al. 2011; Gabor \& Bournaud 2013) and enhance the AGN feedback to keep the galaxies quiescent at earlier times. The cosmological simulation IllustrisTNG (Pillepich et al. 2018) also suggests that smaller star-forming galaxies terminate their star formation activities earlier (Genel et al. 2018).

\subsubsection{Fast Process, PSB Galaxies with Various Sizes}

The other types of mechanisms involve more violent events. Galaxy mergers, interactions, or violent disk instability can efficiently funnel gas into the centers of galaxies, inducing intense star formation in the galaxy centers and exhausting available gas in a short period of time (Yang et al. 2008; Dekel et al. 2009; Zolotov et al. 2015). In addition, AGNs triggered by infalling gas and strong supernova feedback after the starburst could eject ISM from galaxies, accelerating the quenching process (Efstathiou 2000; Springel et al. 2005; Hopkins et al. 2006, 2008). The merger can also enhance turbulence and make the gas stable against gravitational collapse (Ellison et al. 2018). The rapidly rising SFR and the subsequent quenching process can happen within a few hundreds of Myr. In this scenario, the last period of star formation can build up a dense core in the centers. The descendant galaxy can thus have a smaller $R_{e}$ than its 


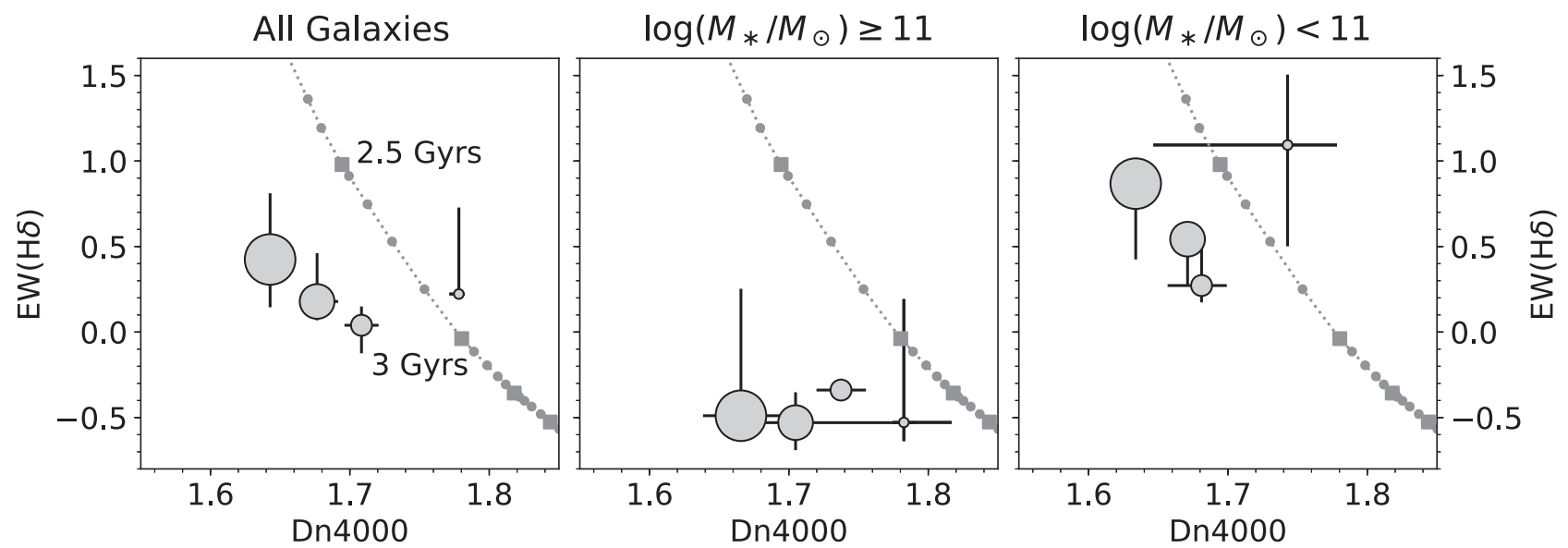

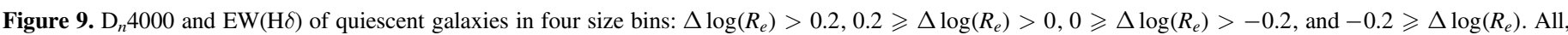

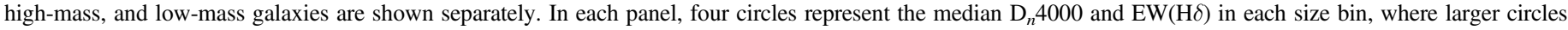

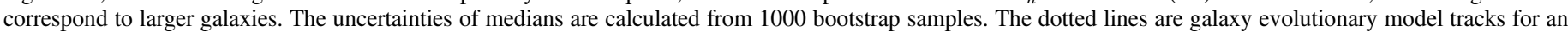

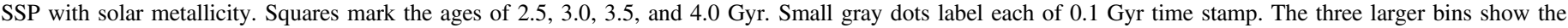
correlation between the ages and the sizes; larger galaxies are on average younger. Nevertheless, this correlation is less significant for massive galaxies.

progenitor (Barnes \& Hernquist 1991; Mihos \& Hernquist 1994; Barnes \& Hernquist 1996; Bournaud et al. 2011; Dekel \& Burkert 2014; Zolotov et al. 2015).

Recent works have identified candidate progenitors of compact PSB galaxies. Case studies on some compact starforming galaxies at $z \sim 2$ found that they have centrally concentrated cores in rest-frame UV and optical wavelengths and short gas depletion times (Barro et al. 2016, 2017; Popping et al. 2017), showing that these galaxies are possibly building the central dense cores and rapidly exhausting the gas reservoir simultaneously. After star formation stops, the stellar population is dominated by A-type stars and the quiescent descendants would be classified as compact PSB galaxies.

\subsubsection{Coexistence of Multiple Pathways to Quiescence}

Our results suggest strongly that there are at least two types of mechanisms with distinct timescales in effect. Neither of the processes discussed above alone can easily produce the complex relationships among the size, the ages, and the recent star formation history.

First of all, simply cutting off the supply of cold gas cannot produce PSB galaxies. Comparisons of the cold gas content and the SFRs of star-forming galaxies at $z<1$ generally show that the gas depletion timescale is of the order of $1 \mathrm{Gyr}$ or longer (Leroy et al. 2008; Schiminovich et al. 2010; Bigiel et al. 2011; Saintonge et al. 2011; Schruba et al. 2011; Huang et al. 2012; Tacconi et al. 2013; Genzel et al. 2015; Tacconi et al. 2018). Galaxies with such star formation histories unlikely go through the PSB phase. When the SFR becomes low enough that galaxies are classified as quiescent, the stellar populations have already aged and do not exhibit strong Balmer absorption (Le Borgne et al. 2006). To produce PSB galaxies, a much shorter quenching timescale $(\lesssim$ a few hundred Myr) or an additional starburst is necessary (Le Borgne et al. 2006; Wild et al. 2009; Snyder et al. 2011). Moreover, the sizes of PSB galaxies are significantly smaller than those of star-forming galaxies of the same mass (Figures 10 and 11). To transform a star-forming galaxy to a PSB galaxy, both the SFR and the size must be altered by the quenching mechanism.

Second, PSB galaxies are unlikely the progenitors of the majority of the quiescent population, at least up to $z \sim 1$. If the size distribution of PSB galaxies is representative for all new quiescent galaxies, there would not be the age-size correlation that large galaxies are typically younger than smaller galaxies.

The complex correlation among sizes, ages, and recent star formation histories warrants multiple mechanisms transforming galaxies from star-forming to quiescent (also see Schawinski et al. 2014; Belli et al. 2015; Maltby et al. 2018). Figure 12 illustrates these two different processes on the mass-size plane. The red and blue shaded areas represent star-forming and quiescent populations, respectively. The arrows show how galaxies evolve on the mass-size plane relative to the bulk of the population when star-forming galaxies start to leave the main sequence to the time they are defined as quiescent.

Galaxies going through the slow process do not change their stellar masses and sizes much along with the SFRs (Figure 12(a)). Galaxies can grow slightly in mass and size due to the residual star formation (Tacchella et al. 2015, 2018). Meanwhile, after the star-forming disk fades, the $R_{e}$ measured in optical wavelengths would become smaller because the light from star-forming galaxies is dominated by the disk component, which is more extended than the underlying mass profile (Szomoru et al. 2013; Lang et al. 2014; Mosleh et al. 2017). Empirically, this change in size is found to be in general small (Szomoru et al. 2013; Mosleh et al. 2017). Overall, the newly formed quiescent galaxies have masses and sizes similar to their progenitor star-forming galaxies and larger than pre-existing, older quiescent galaxies.

On the other hand, mechanisms that shut off the star formation rapidly ( $<$ a few hundred Myr) can produce quiescent galaxies much smaller than their progenitor starforming galaxies. The mass increases due to intense star formation. The size, quantified as $R_{e}$, can become a few times smaller because new stars form in the center of the galaxy (Hopkins et al. 2013; Wellons et al. 2015; Tacchella et al. 2016). The amount of change in mass and size depends on the properties of the progenitor (Hopkins et al. 2013; Dekel \& Burkert 2014; Blumenthal \& Barnes 2018).

\subsubsection{The Relative Importance of Different Routes}

The relative importance of different mechanisms likely depends on several factors. In the early universe, the merger rate is higher (Hopkins et al. 2010; Lotz et al. 2011; 


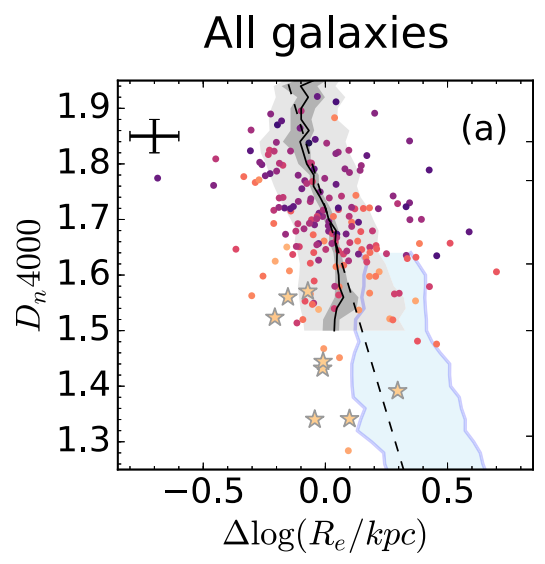

$$
\log \left(M_{*} / M_{\odot}\right) \geq 11
$$

$\log \left(M_{*} / M_{\odot}\right)<11$
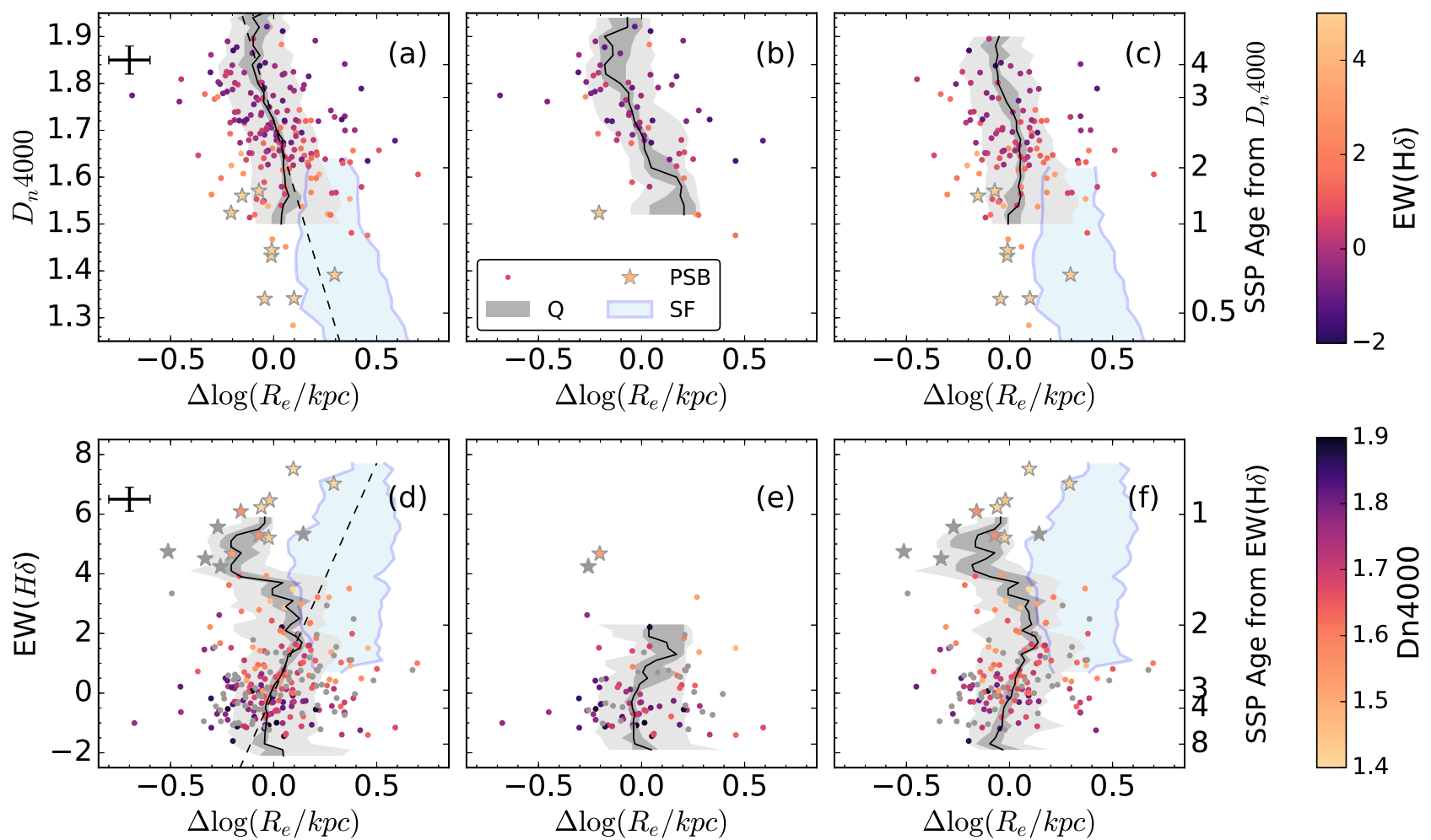

Figure 10. Sizes as a function of $\mathrm{D}_{n} 4000$ and $\mathrm{EW}(\mathrm{H} \delta$ ) of all (left), high-mass (center), and low-mass (right) quiescent galaxies. PSB galaxies are labeled by stars. In panels (a)-(c), the colors show the $\mathrm{EW}\left(\mathrm{H} \delta\right.$ ) value. In panels (d)-(f), the colors show the $\mathrm{D}_{n} 4000$, and galaxies without $\mathrm{D}_{n} 4000$ measurements are in gray. The data points are the same as in Figure 8. The solid black lines show the running median of $\Delta \log \left(R_{e}\right)$, at fixed $\mathrm{D}_{n} 4000$ or EW $(\mathrm{H} \delta)$. The light gray areas indicate the 16th and 84th percentiles of the distribution at fixed indices. The dark gray areas are the uncertainties of the median $\Delta \log \left(R_{e}\right)$, estimated from 1000 bootstrap samples. The light blue area shows the 16th and the 84th percentiles of the $\Delta \log \left(R_{e}\right)$ of star-forming galaxies for comparison. The $y$-axis on the right-hand side labels the corresponding ages of a solar-metallicity SSP of each index. We only show intervals with more than five galaxies in bins of 0.05 in $\mathrm{D}_{n} 4000$ and $0.8 \AA$ in $\mathrm{EW}(\mathrm{H} \delta)$. There are too few massive star-forming galaxies to plot. The dashed lines in panels (a) and (d) show the best-fit relations between $\Delta \log \left(R_{e}\right)$ and the indices at $\mathrm{D}_{n} 4000 \geqslant 1.6$ and $\mathrm{EW}(\mathrm{H} \delta) \leqslant 2 \AA$ (Equation (3)) and the extrapolation beyond the fitting ranges. The quiescent and star-forming galaxies form a continuous sequence on the $\mathrm{D}_{n} 4000-\Delta \log \left(R_{e}\right)$ plane. As $\mathrm{D}_{n} 4000$ decreases, the median size increases. EW(H $\delta$ ) shows qualitatively same behavior, except for galaxies with the highest $\mathrm{EW}(\mathrm{H} \delta)$. PSB galaxies are much smaller than star-forming galaxies with the same $\mathrm{EW}(\mathrm{H} \delta)$.

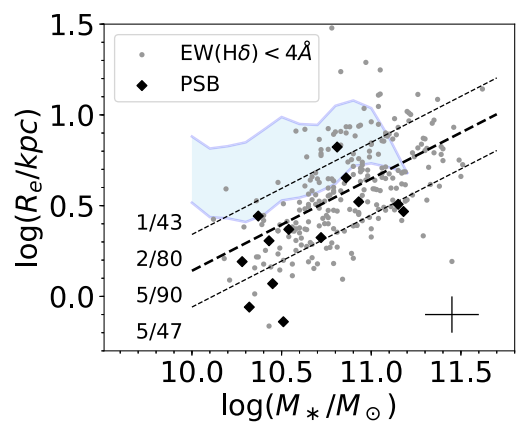

Figure 11. Distribution of PSB galaxies on the mass-size plane. Big black diamonds are quiescent galaxies with $\mathrm{EW}(\mathrm{H} \delta) \geqslant 4 \AA$, defined as PSB galaxies. The small gray dots are the other quiescent galaxies. The thick dashed line is the best-fit mass-size relation. The thin dashed lines label the \pm 0.2 dex $(\sim 1 \sigma)$ range around the best-fit relation. The blue shaded area shows the 16th and 84th percentiles of the sizes of star-forming galaxies at fixed stellar mass. The numbers of all quiescent galaxies and PSB galaxies in four different ranges of sizes are labeled at the left. The majority of PSB galaxies (10/13) are smaller than the average size of quiescent galaxies at fixed stellar mass. About $40 \%$ of PSB galaxies (5/13) are located below the $1 \sigma$ distribution. Furthermore, most PSB galaxies are significantly smaller than star-forming galaxies.

Xu et al. 2012; Rodriguez-Gomez et al. 2015; Man et al. 2016), and galaxies are gravitationally unstable due to high gas fraction; rapid gas inflows and catastrophic events are more common (Dekel et al. 2009; Dekel \& Burkert 2014; Zolotov et al. 2015).
Empirically, the abundance of massive PSB galaxies increases along with redshifts up to $z \sim 2$ (Tran et al. 2004; Yan et al. 2009; Vergani et al. 2010; Wu et al. 2014; Wild et al. 2016; Maltby et al. 2018), which also suggests that the star formation in galaxies is more often shut off rapidly at higher redshifts.

The environment is another relevant factor. The fraction of PSB galaxies is larger in galaxy groups and clusters (Tran et al. 2003; Poggianti et al. 2009; Muzzin et al. 2012; Dressler et al. 2013; Wu et al. 2014; Paccagnella et al. 2018). The merger rates are higher in denser regions (Sobral et al. 2011). Moreover, in galaxy clusters, interstellar gas may be removed by interaction with dense intracluster medium, known as ram pressure stripping (Gunn et al. 1972). The ram pressure is weak outside galaxy groups and clusters but is considered an indispensable mechanism in order to explain the high PSB fractions in galaxy clusters (Muzzin et al. 2014; Wu et al. 2014; Paccagnella et al. 2018). Only one PSB galaxy in our sample is a candidate cluster member (according to the catalog of X-ray clusters in the COSMOS field; Finoguenov et al. 2007). Our conclusion in this paper should apply to only field galaxies.

In addition, at low stellar masses, galaxies are prone to environmental effects and AGN and stellar feedback. Extra quenching mechanisms may be in effect and produce low-mass PSB galaxies (Maltby et al. 2018). 

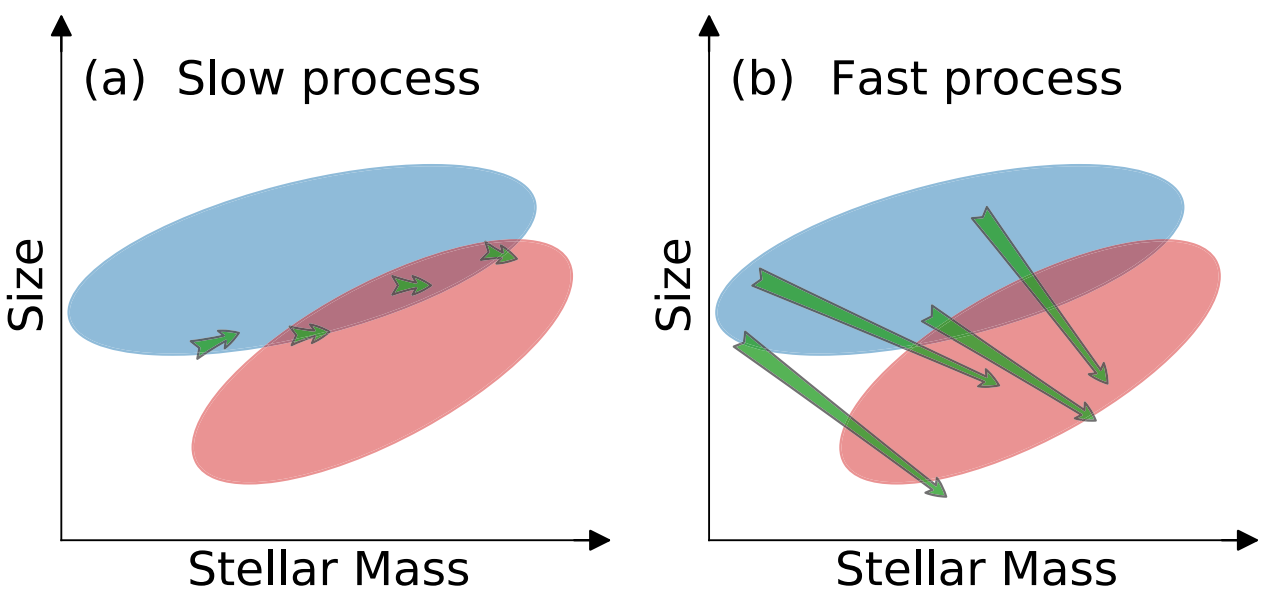

Figure 12. Evolution of the slow and the fast process on the mass-size plane. The blue and red shaded areas represent star-forming and quiescent populations, respectively. The arrows show how galaxies evolve on the mass-size plane relative to the bulk of the population when star-forming galaxies start to leave the main sequence to the time they are defined as quiescent. (a) Galaxies going through the slow process do not significantly change their masses and sizes. The stellar masses become slightly larger as the residual cold gas turns into stars. Although galaxies grow, the sizes measured in optical light may become slightly smaller after the extended star-forming disks fade. New quiescent galaxies are thus larger than the bulk of the quiescent population. (b) Galaxies going through the fast process can change their masses and sizes significantly. The masses increase due to episodes of intense star formation. The sizes, quantified as $R_{e}$, decrease because the newly formed stars are centrally concentrated. Depending on the masses and the spatial distribution of newly formed stars, new quiescent galaxies can be smaller than the existing quiescent population.

In reality, the evolutionary tracks of galaxies will not cleanly separate into slow and fast tracks but can fall anywhere in between. Regardless of the specific characteristics of any slow and fast tracks, our data unequivocally show that the relationship between star formation history and structural evolution is complex and can vary strongly from one galaxy to another.

\subsection{Systematic Uncertainties}

\subsubsection{The Effect of Metallicity}

Both $\mathrm{D}_{n} 4000$ and $\mathrm{EW}(\mathrm{H} \delta)$ depend on not only ages but also metallicities. In this paper, we interpret the dependence of indices on size as an effect of the age, assuming galaxies of the same masses have the same metallicity. In reality, the stellar mass-stellar metallicity relation of quiescent galaxies at $z \sim 0.7$ shows a scatter of 0.16 dex (Gallazzi et al. 2014; see also Jørgensen et al. 2017). The dependence of indices on size may alternatively be an effect of metallicity if the sizes and the metallicity are also correlated. Such a correlation has been observed in the local universe (McDermid et al. 2015; Wu et al. 2015; Scott et al. 2017; Barone et al. 2018; Li et al. 2018) but not yet at higher redshifts.

Here we examine whether the variations in $\mathrm{D}_{n} 4000$ and $\mathrm{EW}(\mathrm{H} \delta)$ can be attributed to variations in metallicity (instead of age). We test the most extreme scenario in which the scatter in the mass-size relation corresponds one-to-one to the scatter in the mass-stellar metallicity relation.

We assign a fiducial metallicity to each galaxy based on the size:

$$
[Z / H]=-0.8 \times \Delta \log \left(R_{e}\right) .
$$

This is based on the assumption that the 0.16 dex scatter in the metallicity is entirely due to the variation in the size $(0.2 \mathrm{dex}$ scatter; Section 2.5), and a galaxy with the median size has solar metallicity. We assign the same stellar age of $3 \mathrm{Gyr}$ to all galaxies and produce mock spectra using Bruzual \& Charlot (2003) SSP models then measure their $\mathrm{D}_{n} 4000$ and $\mathrm{EW}(\mathrm{H} \delta)$.

Figure 13 shows how the $\mathrm{D}_{n} 4000$ and $\mathrm{EW}(\mathrm{H} \delta)$ of the mock spectra vary with the size compared to the observed

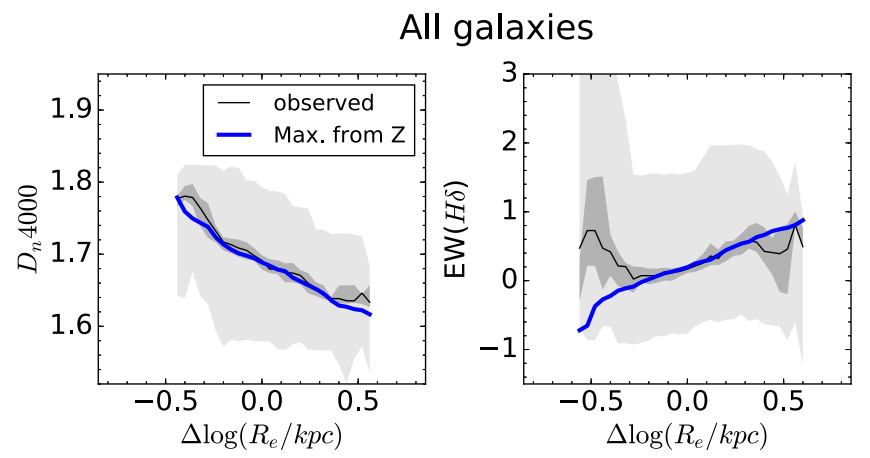

Figure 13. Testing the effect of metallicity on $\mathrm{D}_{n} 4000$ and $\operatorname{EW}(\mathrm{H} \delta)$. The thin black lines and gray shaded areas are the same as Figure 8: the measured median indices, the uncertainties of the medians, and the 16th and 84th percentiles of the distributions. The thick blue lines are $\mathrm{D}_{n} 4000$ and $\mathrm{EW}(\mathrm{H} \delta)$ measured from mock spectra with a fixed age of $3 \mathrm{Gyr}$ and a size-dependent metallicity $[Z / H]=-0.8 \times \Delta \log \left(R_{e}\right)$. The thick blue lines represent the maximum effect due to a size-dependent metallicity (see detailed discussion in Section 4.3.1). The metallicity produces a comparable size dependence only in the most extreme case shown here.

correlations. The similarity is striking, which immediately reveals that metallicity variation can play an important role. However, it is unlikely that the correlation between the size and the metallicity has no intrinsic scatter at all. The general trend we find in Section 3.2 that larger galaxies are younger than smaller galaxies is robust in a qualitative sense. But the metallicity dependence shows that the true correlation may be even weaker than what we find while assuming a fixed, solar metallicity.

Assuming the same $3 \mathrm{Gyr}$ population, the metallicity needs to be $[\mathrm{Z} / \mathrm{H}]<-1$ to have $\mathrm{EW}(\mathrm{H} \delta)>4 \AA$, according to the model of Thomas et al. (2011). This metallicity is extremely low in comparison to the average $([Z / H] \simeq 0.0 \pm 0.16)$ and has not yet been measured in galaxies at similar (Gallazzi et al. 2014; Jørgensen et al. 2017) or even higher redshifts ( $z \sim 3$; Sommariva et al. 2012; Marques-Chaves et al. 2018). It is thus unlikely that the strong Balmer absorption in PSB galaxies is entirely due to low metallicities. 


\subsubsection{The Effect of Age Gradient and Slit Loss}

Our spectra are obtained with slits of $1^{\prime \prime}$ width, which corresponds to $\sim 7 \mathrm{kpc}$ at $z \sim 0.7$. For large face-on galaxies or those misaligned with slits, the spectra miss light from galaxy outskirts, which likely consist of younger stellar populations (Szomoru et al. 2011; González Delgado et al. 2015; Goddard et al. 2017; Wang et al. 2018). The age obtained from the spectra may be thus biased old.

We test the potential bias using a subset of spectra that is spatially resolved. We select 152 larger quiescent galaxies $\left(R_{e}>0\right.$ ".5) whose major axes align with the slits $(\Delta$ P.A. $<$ $\left.45^{\circ}\right)$ or are face-on $(b / a>0.7)$. We measure the $\mathrm{D}_{n} 4000$ and $\mathrm{EW}(\mathrm{H} \delta)$ from the central $1^{\prime \prime}$ along the slit direction. The central $\mathrm{D}_{n} 4000$ is on average $0.05 \pm 0.01$ larger than when integrating over the entire galaxy, suggesting that the centers of galaxies are older. On the other hand, the difference in $\operatorname{EW}(\mathrm{H} \delta)$ is consistent with zero, $0.02 \pm 0.03 \AA$. We conclude that our result in Section 3.2, namely that on average larger galaxies are younger, is qualitatively not affected and in fact would be strengthened by the bias introduced by the finite slit width.

We also confirm that the selection of PSB galaxies is not biased in size; there is only one galaxy that would be defined as a PSB galaxy according to the integrated spectrum but not the central spectrum. Missing light at the outskirts of large galaxies does not result in a bias in the sizes of the PSB sample.

\subsubsection{Stellar Mass-to-light Ratio of PSB Galaxies}

We measure the sizes using HST F814W images, which correspond to the rest-frame $B$-band for galaxies at $z \sim 0.7$. The light of PSB galaxies is dominated by A-type stars, which may not necessarily trace the mass profiles well if the last episode of star formation has a distinct spatial distribution from old stellar populations.

A few works have studied the sizes of PSB galaxies using HST F160W images but focusing on galaxies mainly at $z>1$, sampling the redder part at the rest-frame optical regime (Belli et al. 2015; Yano et al. 2016; Almaini et al. 2017). These studies generally find that PSB galaxies are $\sim 0.2$ dex smaller than average quiescent galaxies. A more reliable size should be measured at longer rest-frame wavelengths, preferentially in rest-frame near-IR. The new "drift and shift" observing mode of the HST WFC3 IR channel allows for a much faster survey speed (Momcheva et al. 2017). We have obtained the F160W images of a large fraction of our sample and will examine the structures of galaxies in multiple wavelengths. For PSB galaxies at $z>1$, it will require JWST to provide images at longer wavelengths.

\section{Summary}

In this paper, we present the size and age-sensitive spectral features $\left(\mathrm{D}_{n} 4000\right.$ and $\left.\mathrm{EW}(\mathrm{H} \delta)\right)$ of 467 quiescent galaxies with stellar masses $M_{*}>10^{10} M_{\odot}$ at $z \sim 0.7$. We measure the sizes from HST F814W images and age indicators using ultra-deep spectra from the LEGA-C survey. The high-quality spectra allows us to measure age indicators accurately for individual galaxies.

At fixed stellar mass, $\mathrm{D}_{n} 4000$ and $\mathrm{EW}(\mathrm{H} \delta)$ show large individual variations, distributed over a wide range of $\mathrm{D}_{n} 4000 \simeq$ 1.6-1.8 and $\mathrm{EW}(\mathrm{H} \delta) \simeq-1 \AA-2 \AA$, respectively. These ranges correspond to an age range of $\sim 1.5 \mathrm{Gyr}$ for a solar-metallicity SSP. Nevertheless, we find a weak trend that larger galaxies have on average smaller $\mathrm{D}_{n} 4000$ and larger $\mathrm{EW}(\mathrm{H} \delta)$. Excluding the most compact galaxies, the variation in median $\mathrm{D}_{n} 4000$ and $\mathrm{EW}(\mathrm{H} \delta)$ is $\sim 0.06$ and $\sim 0.5 \AA$, respectively. The corresponding age variation is $<500 \mathrm{Myr}$. On the contrary, quiescent galaxies with rapidly declining SFRs (PSB galaxies) do not follow the general age-size correlation; they are among the youngest and are much smaller than other young quiescent galaxies.

These two seemingly contradictory statements are often presented separately in the literature and raise some dispute regarding the formation and evolution of quiescent galaxies. We demonstrate that both observations are correct, and only a large sample of galaxies with high-quality spectra can reveal the complex correlation. While bulk of the population have $\mathrm{D}_{n} 4000$ and $\mathrm{EW}(\mathrm{H} \delta)$ that suggest younger galaxies are on average larger, there is a small fraction of galaxies with elevated $\mathrm{H} \delta$ absorption, indicating a rapidly quenching star formation history, that are smaller than the average.

This correlation suggests that there are multiple evolutionary pathways to quiescence. Star-forming galaxies that slowly exhaust their cold gas reservoirs join the large side of the red sequence. These galaxies do not go through a PSB phase because the star formation fades in a longer timescale. They can drive at least partially the size evolution of quiescent galaxies and produce the progenitor bias. On the other hand, violent events such as galaxy mergers or violent disk instabilities can produce PSB galaxies that are much smaller than their starforming progenitors and even smaller than the existing quiescent population. The star formation stops within a few hundred Myr, accompanied by structural changes that shrink the sizes of galaxies.

Based on observations made with ESO Telescopes at the La Silla Paranal Observatory under programme ID 194-A.2005 (the LEGA-C Public Spectroscopy Survey). We thank the referee for the valuable comments. This project has received funding from the European Research Council (ERC) under the European Unions Horizon 2020 research and innovation programme (grant agreement No. 683184). C.S. acknowledges support from the Deutsche Forschungsemeinschaft (GZ: WE 4755/4-1). V.W. acknowledges funding from the ERC (starting grant SEDmorph, PI. Wild).

\section{Appendix \\ Using Different Definitions of Quiescence}

We repeat the analysis in Section 3 using quiescent galaxies selected by the sSFR and UVJ colors. Figure 14 shows the distributions of redshifts and stellar masses of each sample. Comparing to the $\operatorname{EW}(\mathrm{H} \beta)$ selection used in the main text, these two samples contain galaxies with $z \gtrsim 0.8$. The best-fit parameters of the mass-size relation (Figure 15) is listed in Table 2.

We find qualitatively the same conclusions using different definitions of quiescence. More massive galaxies have larger $\mathrm{D}_{n} 4000$ and smaller $\mathrm{EW}(\mathrm{H} \delta)$ (Figures 16, 17). Despite large individual variations, larger quiescent galaxies have on average smaller $\mathrm{D}_{n} 4000$ and larger $\mathrm{EW}(\mathrm{H} \delta)$, indicating younger stellar ages (Figures 18, 19, 20). On the other hand, PSB galaxies are not large quiescent galaxies and much smaller than starforming galaxies (Figure 21). 

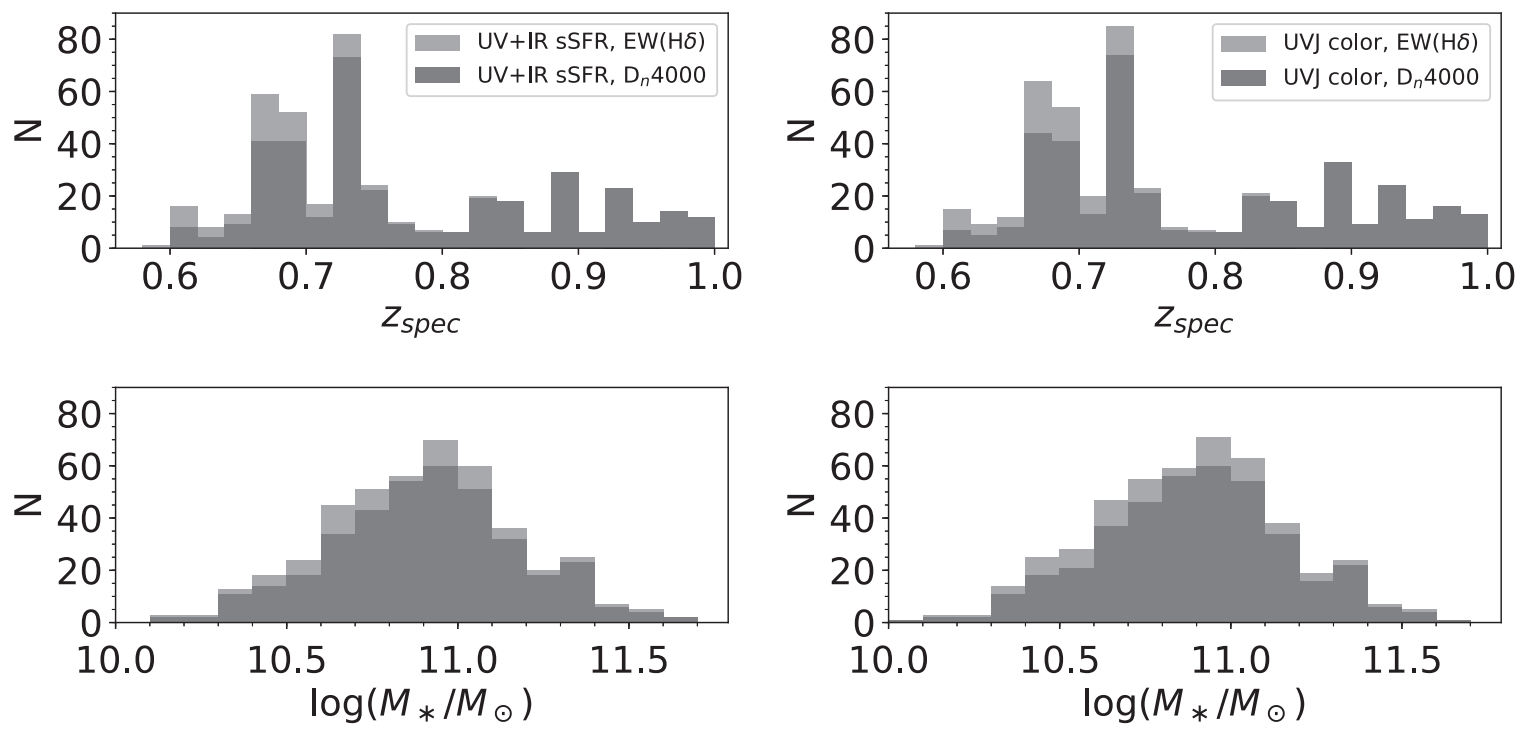

Figure 14. Distributions of redshifts and stellar masses of quiescent galaxies selected based on the sSFR and $U V J$ color, the same as Figure 2. The light and dark gray histograms are the distributions of galaxies with only $\mathrm{EW}(\mathrm{H} \delta)$ and both $\mathrm{EW}(\mathrm{H} \delta)$ and $\mathrm{D}_{n} 4000$ measurements, respectively. These two selections contain more galaxies at $z \gtrsim 0.8$ compared to the $\mathrm{EW}(\mathrm{H} \beta)$ selection.
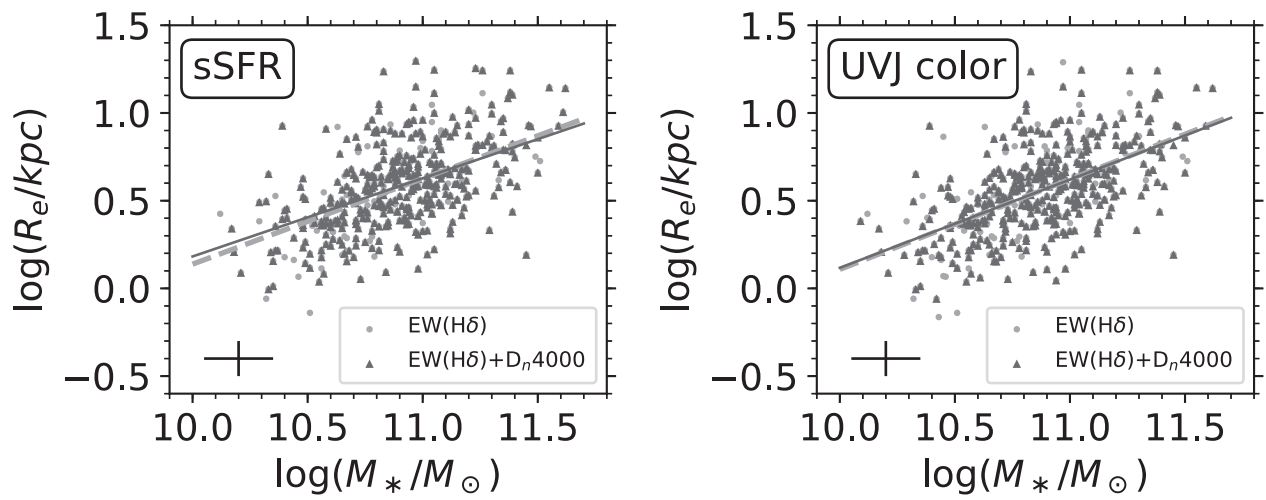

Figure 15. Size and stellar masses of quiescent galaxies selected based on the sSFR and $U V J$ color, the same as Figure 3. Dark gray triangles are galaxies with spectra covering both $\mathrm{D}_{n} 4000$ and $\mathrm{EW}(\mathrm{H} \delta)$. Light gray circles are galaxies for which only $\mathrm{EW}(\mathrm{H} \delta)$ is measurable. The light gray dashed line and the dark gray solid line are the best-fit mass-size relation for all galaxies and galaxies with $\mathrm{D}_{n} 4000$ measurements, respectively.

Table 2

Best-fit Mass-Size Relation

\begin{tabular}{lcc}
\hline \hline Selection & $\mathrm{a}$ & $\mathrm{b}$ \\
\hline UV+IR sSFR & $0.45_{-0.04}^{+0.05}$ & $0.63_{-0.01}^{+0.01}$ \\
UV+IR sSFR, $\mathrm{D}_{n} 4000$ & $0.46_{-0.06}^{+0.06}$ & $0.63_{-0.01}^{+0.00}$ \\
UVJ color & $0.48_{-0.06}^{+0.05}$ & $0.62_{-0.00}^{+0.00}$ \\
UVJ color, $\mathrm{D}_{n} 4000$ & $0.50_{-0.05}^{+0.04}$ & $0.62_{-0.01}^{+0.00}$ \\
\hline
\end{tabular}

Note. $\log \left(R_{e}\right)=a \times\left[\log \left(M_{*} / M_{\odot}\right)-11\right]+b$. 


\section{UV+IR SSFR}
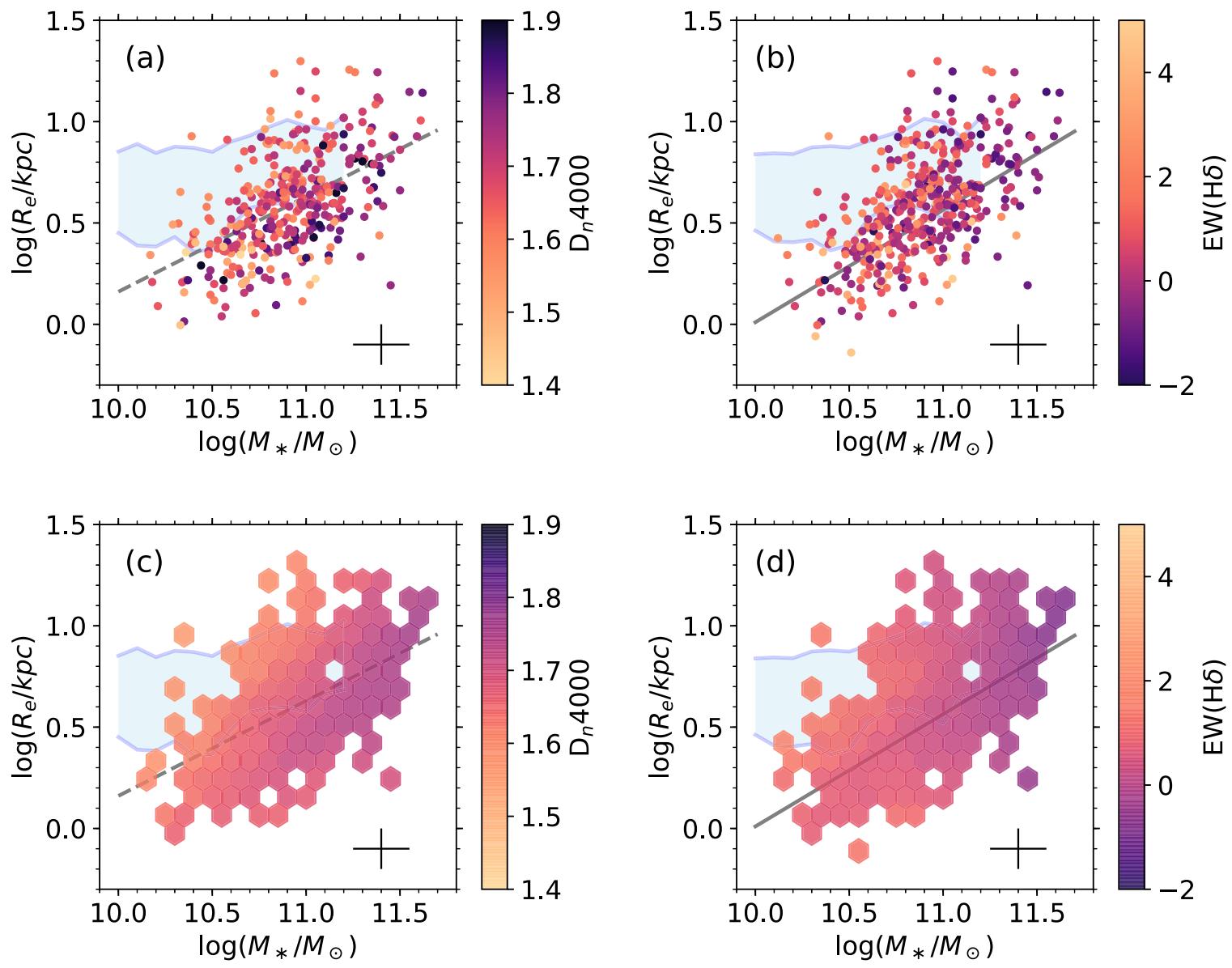

Figure 16. Mass-size relation of quiescent galaxies selected by sSFR, color-coded by the $\mathrm{D}_{n} 4000$ and $\mathrm{EW}(\mathrm{H} \delta)$ of individual galaxies and averaging over nearby data points on the mass-size plane using the LOESS method. The light blue shaded areas are the 16th and 84th percentiles of the sizes of star-forming galaxies at fixed mass. The dashed and solid lines are the best-fit mass-size relations of quiescent galaxies. The cross in the bottom-right corner represents the uncertainties in stellar mass $(0.15$ dex $)$ and sizes $(0.10$ dex $)$ 


\section{UVJ color}
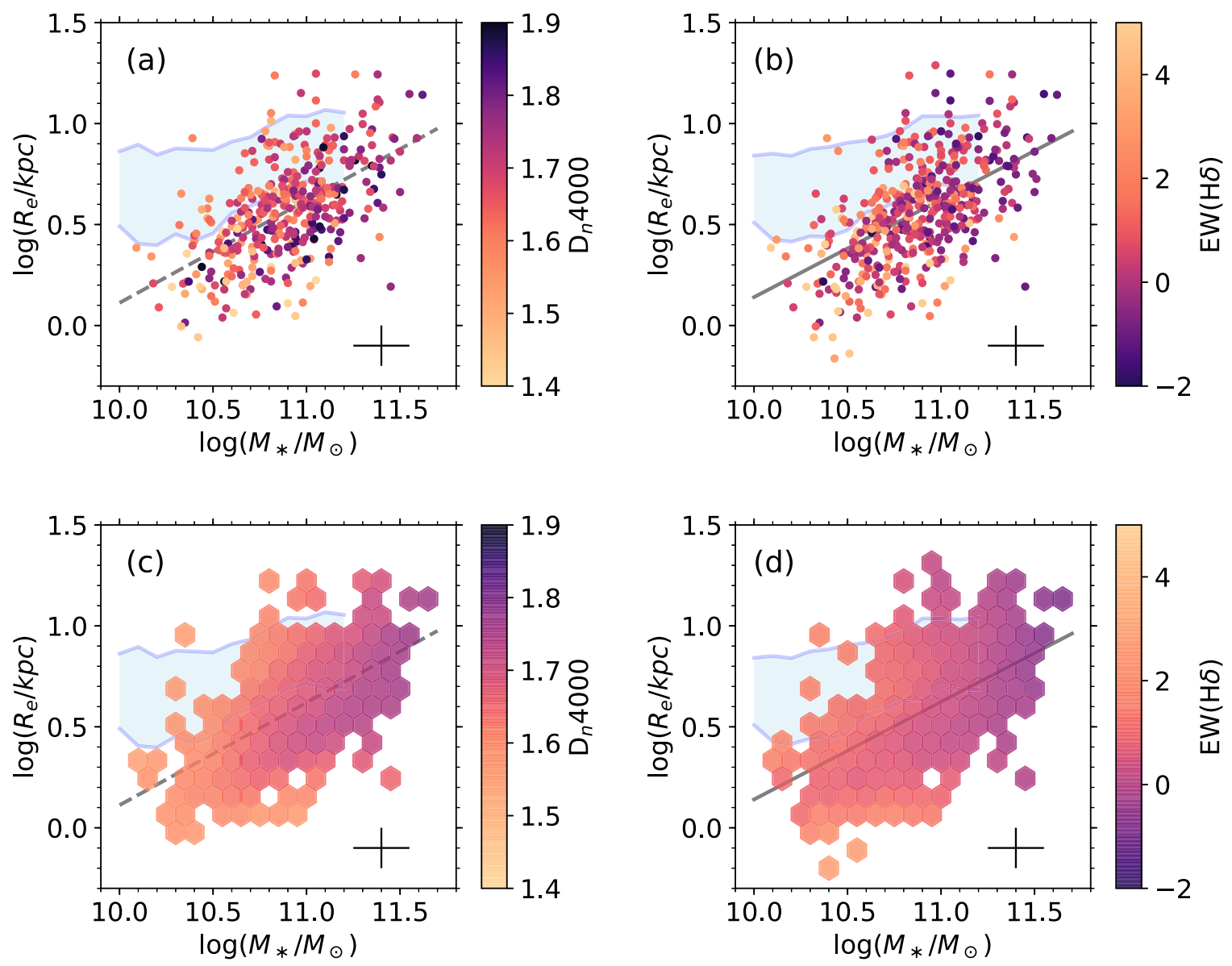

Figure 17. Mass-size relation of quiescent galaxies selected by the $U V J$ colors, color-coded by the $\mathrm{D}_{n} 4000$ and $\mathrm{EW}(\mathrm{H} \delta$ ) of individual galaxies and averaging over nearby data points on the mass-size plane using the LOESS method. The dependence on the size is not clear. The light blue shaded areas are the 16th and 84th percentiles of the sizes of star-forming galaxies at fixed mass. The dashed and solid lines are the best-fit mass-size relations of quiescent galaxies. 
UV+IR SSFR

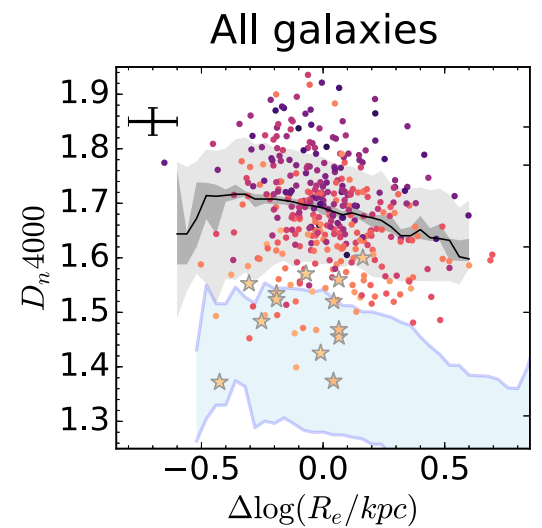

$\log \left(M_{*} / M_{\odot}\right) \geq 11$

$\mathrm{Q} \quad \star \quad \mathrm{PSB} \square \mathrm{SF}$
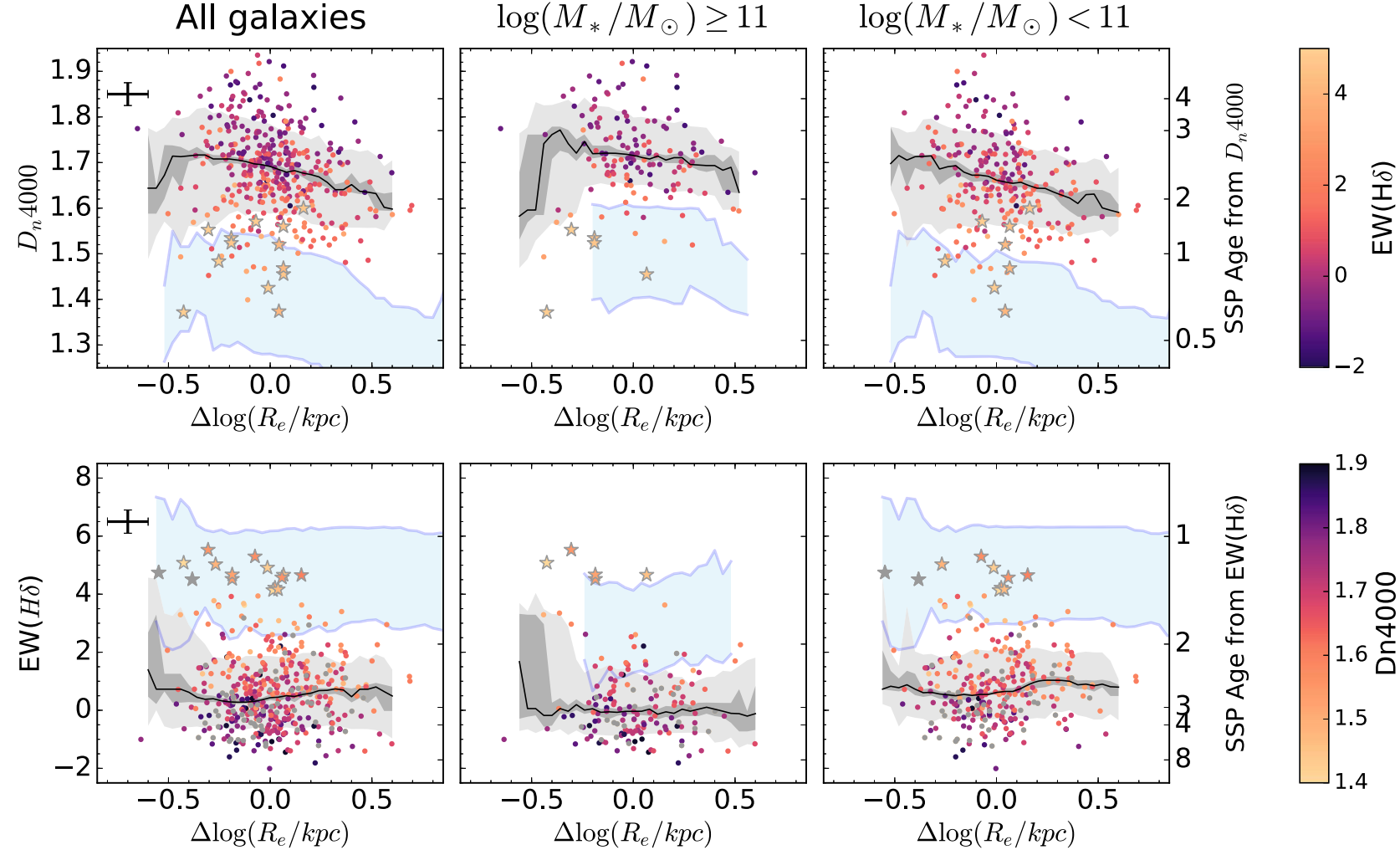

\section{UVJ color $\cdot \quad \cdot \mathrm{Q} \quad * \quad \mathrm{PSB} \quad \square \mathrm{SF}$}
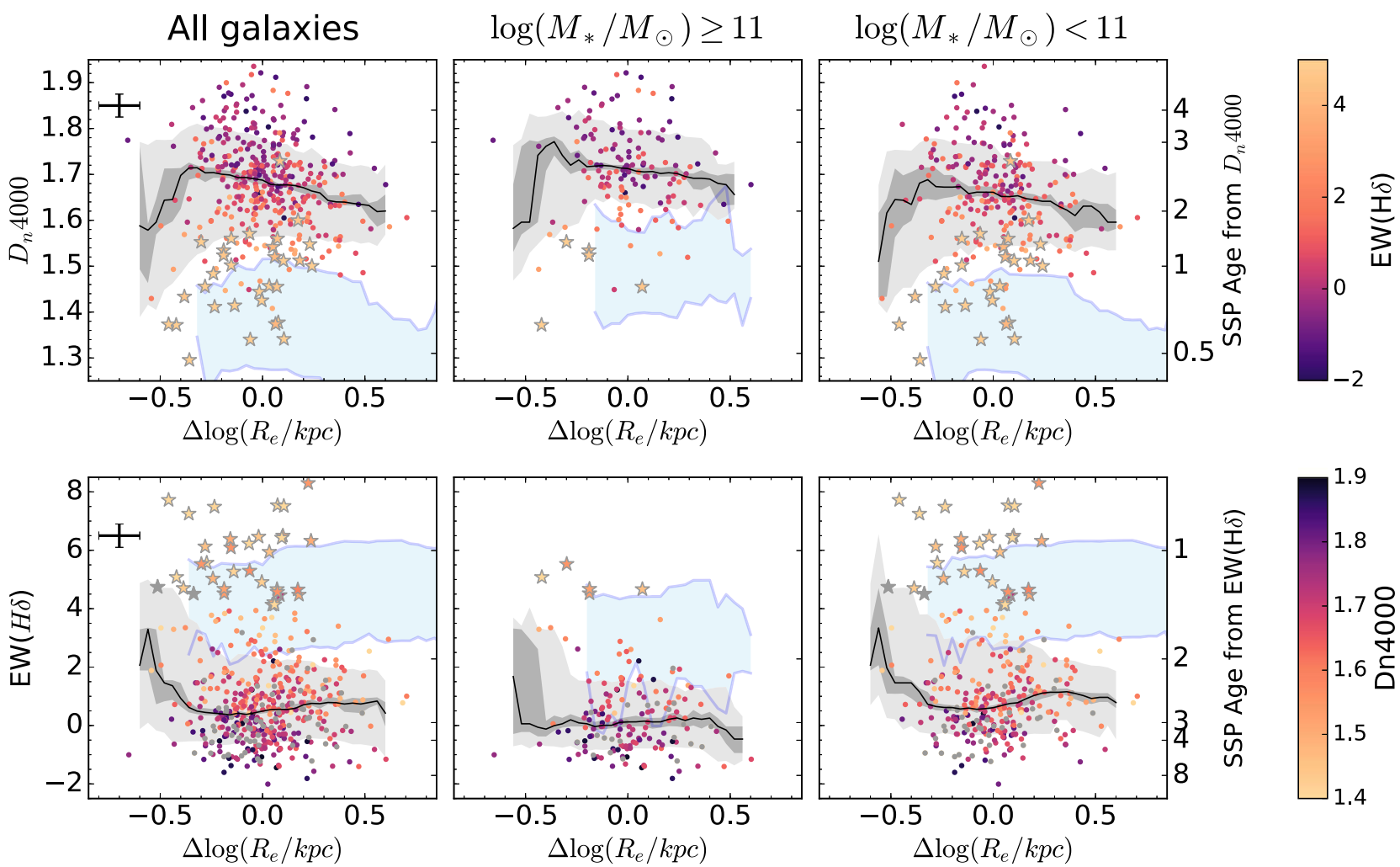

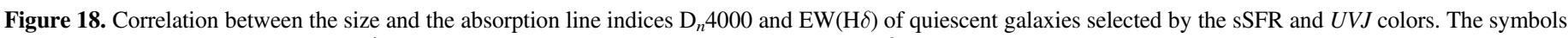

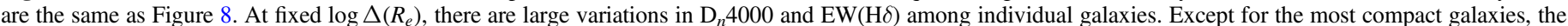
median $\mathrm{D}_{n} 4000$ is smaller for large galaxies, but the $\mathrm{EW}(\mathrm{H} \delta)$ does not show a clear size dependence. 
UV+IR SSFR

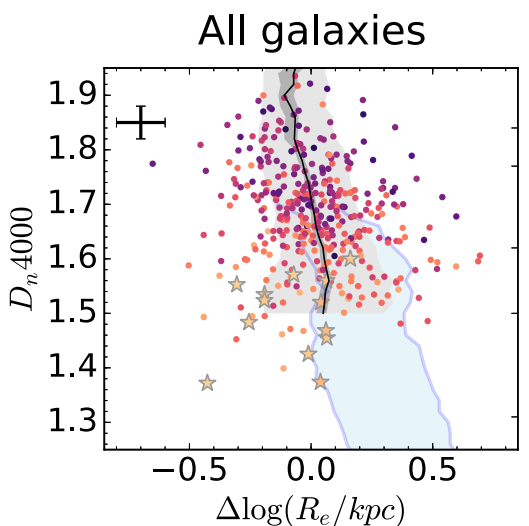

$$
\log \left(M_{*} / M_{\odot}\right) \geq 11
$$

$\log \left(M_{*} / M_{\odot}\right)<11$
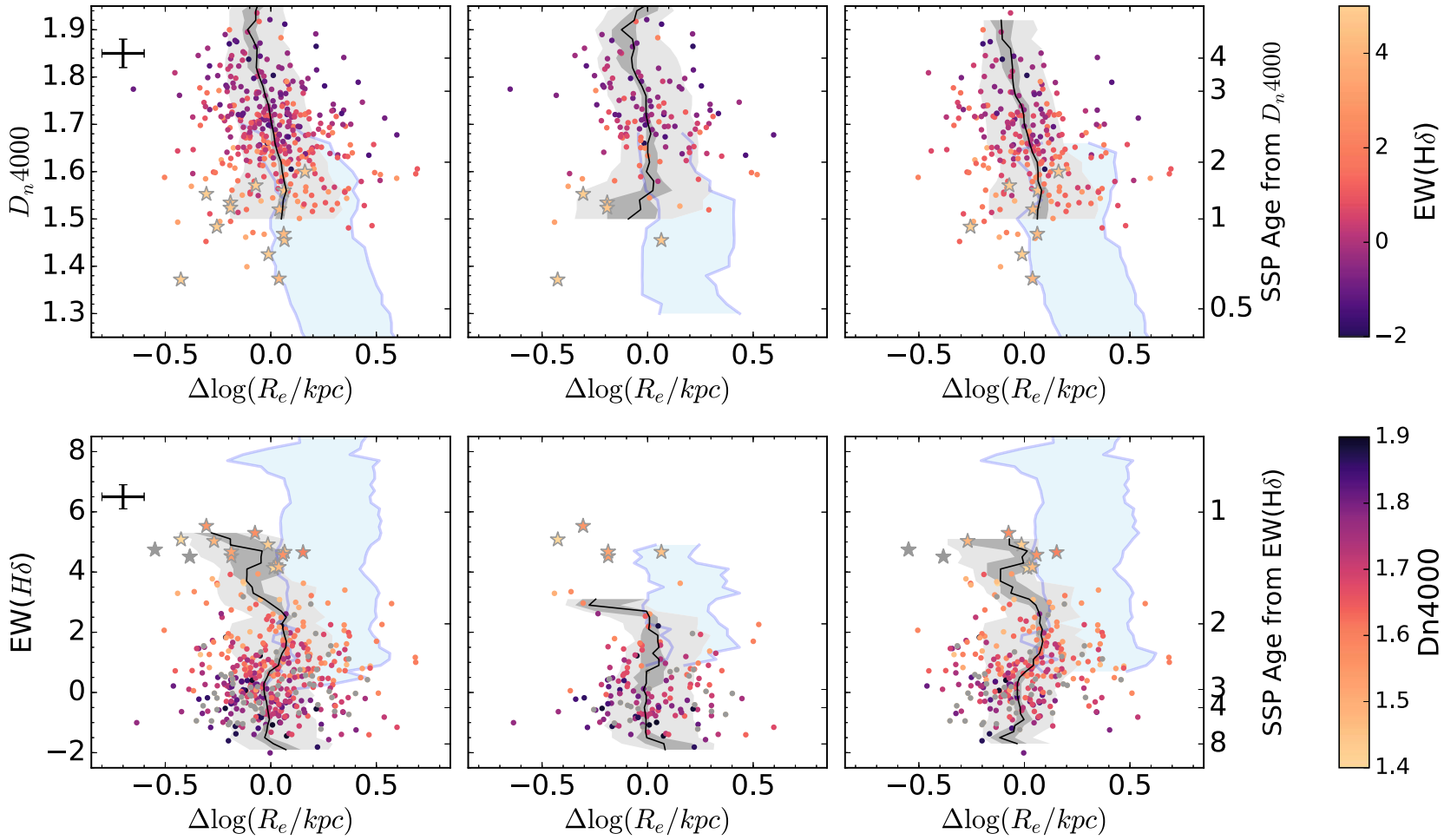

\section{UVJ color}
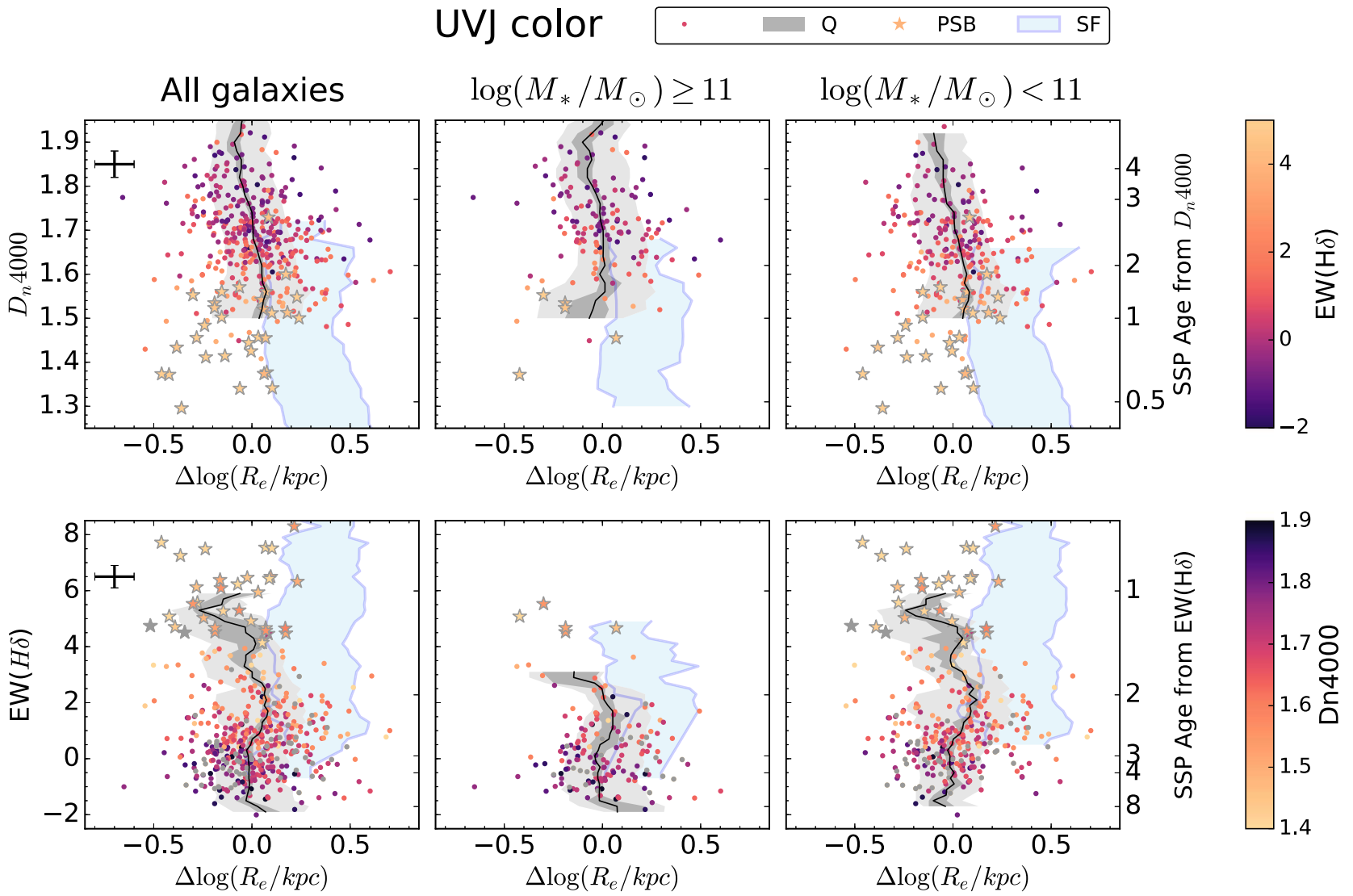

Figure 19. Sizes of quiescent galaxies as a function of $\mathrm{D}_{n} 4000$ and $\mathrm{EW}(\mathrm{H} \delta)$, selected by the sSFR and $U V J$ colors. The symbols are the same as Figure 10 . PSB galaxies are on average smaller than other quiescent galaxies and significantly smaller than star-forming galaxies with similar indices. The median $\log \Delta\left(R_{e}\right)$ of the sSFR and $U V J$ selected PSB galaxies are $-0.07 \pm 0.08$ and $-0.06 \pm 0.07$, respectively. 


\section{All Galaxies}
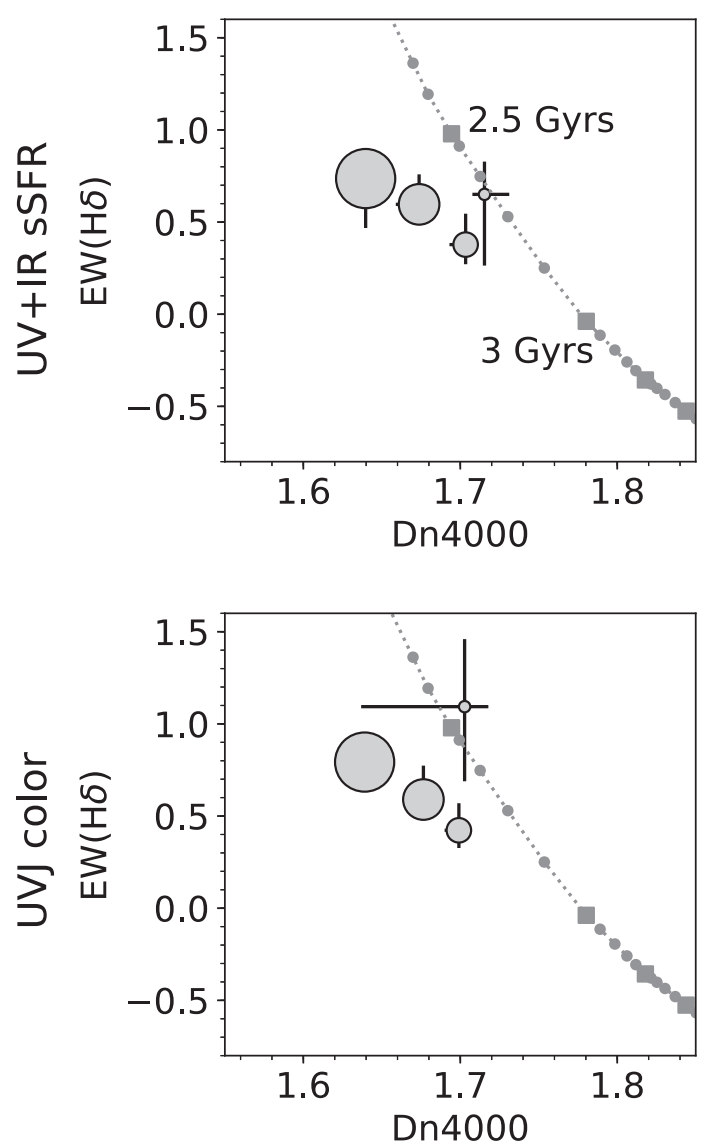

$\log \left(M_{*} / M_{\odot}\right) \geq 11$
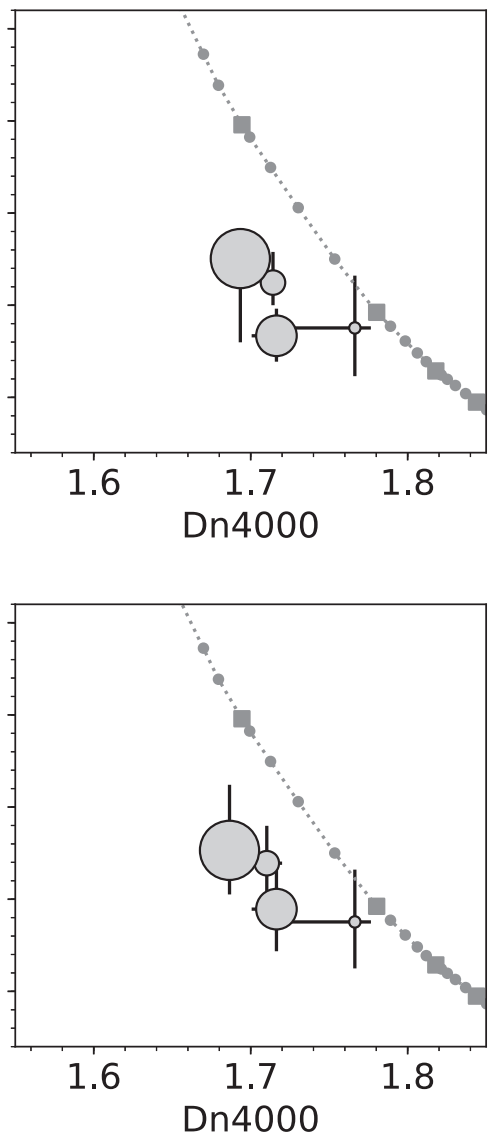

$\log \left(M_{*} / M_{\odot}\right)<11$
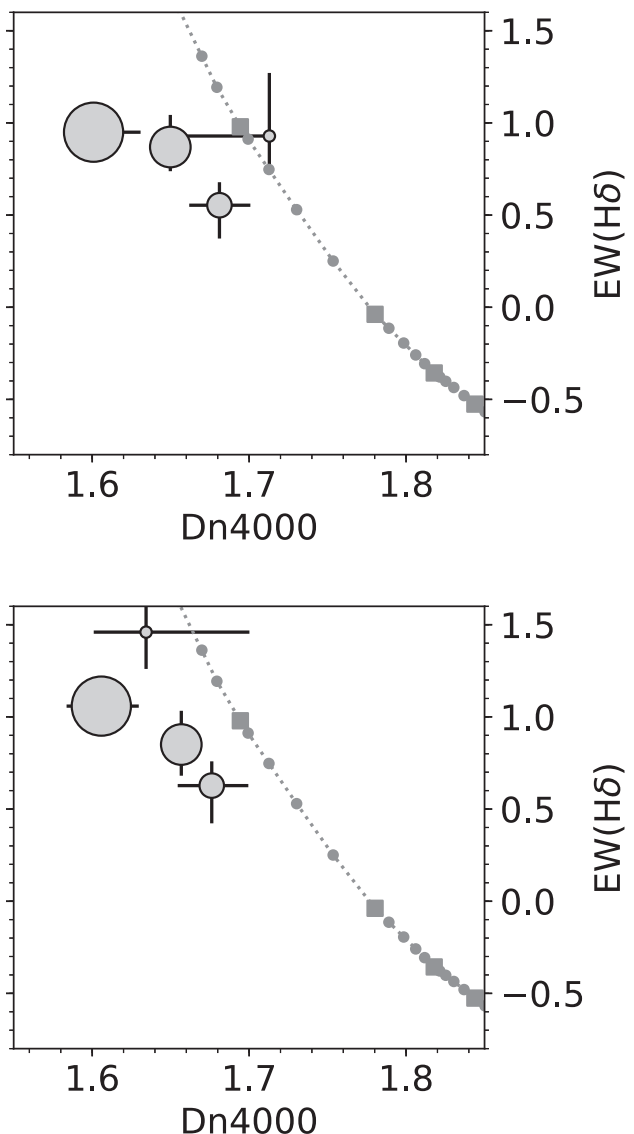

Figure 20. Median $\mathrm{D}_{n} 4000$ and $\mathrm{EW}(\mathrm{H} \delta)$ of quiescent galaxies selected base on sSFR and $U V J$ colors in four size bins: $\Delta \log \left(R_{e}\right)>0.2,0.2 \geqslant \Delta \log \left(R_{e}\right)>0$, $0 \geqslant \Delta \log \left(R_{e}\right)>-0.2$, and $-0.2 \geqslant \Delta \log \left(R_{e}\right)$, the same as in Figure 9. Larger circles represent larger galaxies. The uncertainties of medians are calculated from 1000 bootstrap samples. The dotted lines are galaxy evolutionary model tracks for an SSP with solar metallicity. Squares mark the ages of 2.5, 3.0, 3.5, and 4.0 Gyr. Small gray dots label each of the $0.1 \mathrm{Gyr}$ time stamp. The three larger bins show a weak correlation between the ages and the sizes; larger galaxies are on average younger. There is no such correlation for massive galaxies.

UV+IR SSFR

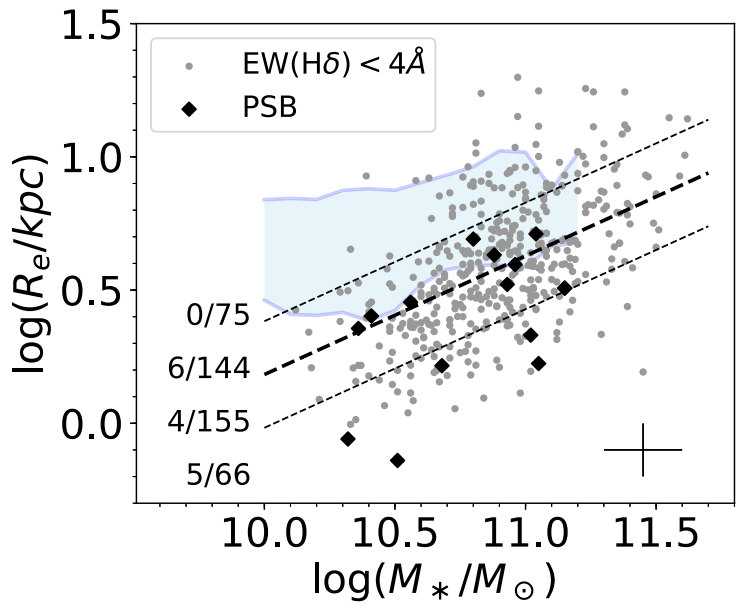

UVJ color

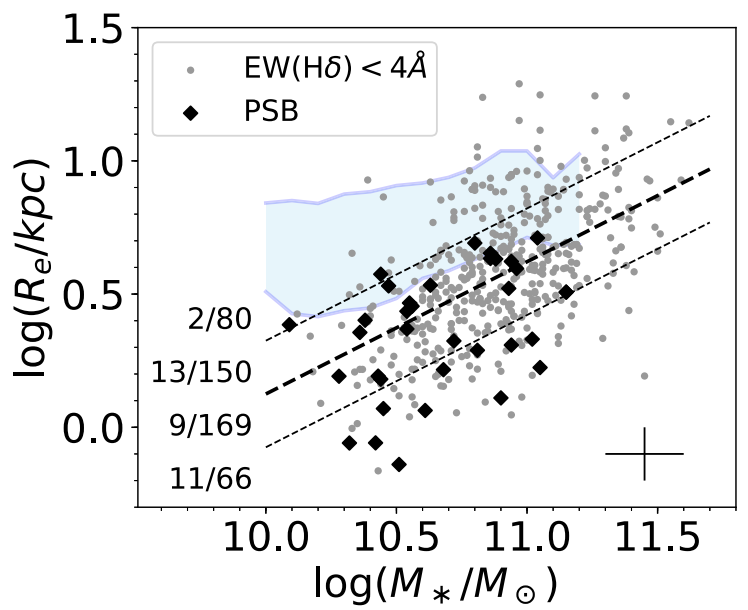

Figure 21. Sizes of PSB galaxies. The "quiescence" is defined by the sSFR or UVJ colors. The symbols are the same as Figure 11 . The numbers of all quiescent galaxies and PSB galaxies in four different ranges of sizes are listed on the left. Most of the PSB galaxies are much smaller than star-forming galaxies. 


\section{ORCID iDs}

Po-Feng Wu (吳柏鋒) (10 https://orcid.org/0000-0002-9665-0440 Arjen van der Wel (i) https://orcid.org/0000-0002-5027-0135 Rachel Bezanson (iD https://orcid.org/0000-0001-5063-8254 Anna Gallazzi (i) https://orcid.org/0000-0002-9656-1800 Camilla Pacifici iib https://orcid.org/0000-0003-4196-0617 Caroline M. S. Straatman (iD https://orcid.org/0000-00015937-4590

Eric F. Bell (ib https://orcid.org/0000-0002-5564-9873 Priscilla Chauke (i) https://orcid.org/0000-0002-1442-984X Marijn Franx (iD https://orcid.org/0000-0002-8871-3026 Adam Muzzin (iD https://orcid.org/0000-0002-9330-9108 David Sobral (iD https://orcid.org/0000-0001-8823-4845

\section{References}

Almaini, O., Wild, V., Maltby, D. T., et al. 2017, MNRAS, 472, 1401 Baldry, I. K., Glazebrook, K., Brinkmann, J., et al. 2004, ApJ, 600, 681 Balogh, M. L., Morris, S. L., Yee, H. K. C., Carlberg, R. G., \& Ellingson, E. 1999, ApJ, 527, 54

Barišić, I., van der Wel, A., Bezanson, R., et al. 2017, ApJ, 847, 72

Barnes, J. E., \& Hernquist, L. 1996, ApJ, 471, 115

Barnes, J. E., \& Hernquist, L. E. 1991, ApJL, 370, L65

Barone, T. M., D'Eugenio, F., Colless, M., et al. 2018, ApJ, 856, 64 Barro, G., Kriek, M., Pérez-González, P. G., et al. 2016, ApJL, 827, L32 Barro, G., Kriek, M., Pérez-González, P. G., et al. 2017, ApJL, 851, L40 Bell, E. F., Wolf, C., Meisenheimer, K., et al. 2004, ApJ, 608, 752 Belli, S., Newman, A. B., \& Ellis, R. S. 2015, ApJ, 799, 206 Best, P. N., Kauffmann, G., Heckman, T. M., et al. 2005, MNRAS, 362, 25 Best, P. N., Ker, L. M., Simpson, C., Rigby, E. E., \& Sabater, J. 2014, MNRAS, 445, 955

Bezanson, R., van der Wel, A., Pacifici, C., et al. 2018, ApJ, 858, 60 Bigiel, F., Leroy, A. K., Walter, F., et al. 2011, ApJL, 730, L13 Blumenthal, K. A., \& Barnes, J. E. 2018, MNRAS, 479, 3952 Bournaud, F., Chapon, D., Teyssier, R., et al. 2011, ApJ, 730, 4 Bruzual, G., \& Charlot, S. 2003, MNRAS, 344, 1000

Calzetti, D., Armus, L., Bohlin, R. C., et al. 2000, ApJ, 533, 682 Cappellari, M. 2017, MNRAS, 466, 798

Cappellari, M., \& Emsellem, E. 2004, PASP, 116, 138

Cappellari, M., McDermid, R. M., Alatalo, K., et al. 2013, MNRAS, 432, 1862 Carollo, C. M., Bschorr, T. J., Renzini, A., et al. 2013, ApJ, 773, 112

Chabrier, G. 2003, PASP, 115, 763

Chauke, P., van der Wel, A., Pacifici, C., et al. 2018, ApJ, 861, 13

Cleveland, W. S., \& Devlin, S. J. 1988, J. Am. Stat. Assoc., 83, 596

Croton, D. J., Springel, V., White, S. D. M., et al. 2006, MNRAS, 365, 11

Dalcanton, J. J., Spergel, D. N., \& Summers, F. J. 1997, ApJ, 482, 659

Dekel, A., \& Birnboim, Y. 2006, MNRAS, 368, 2

Dekel, A., Birnboim, Y., Engel, G., et al. 2009, Natur, 457, 451

Dekel, A., \& Burkert, A. 2014, MNRAS, 438, 1870

Dressler, A., \& Gunn, J. E. 1983, ApJ, 270, 7

Dressler, A., Oemler, Augustus, Jr., Poggianti, B. M., et al. 2013, ApJ, 770, 62

Dressler, A., Smail, I., Poggianti, B. M., et al. 1999, ApJS, 122, 51

Efstathiou, G. 2000, MNRAS, 317, 697

Ellison, S. L., Catinella, B., \& Cortese, L. 2018, MNRAS, 478, 3447

Fabian, A. C. 2012, ARA\&A, 50, 455

Fagioli, M., Carollo, C. M., Renzini, A., et al. 2016, ApJ, 831, 173

Finoguenov, A., Guzzo, L., Hasinger, G., et al. 2007, ApJS, 172, 182

Franzetti, P., Scodeggio, M., Garilli, B., et al. 2007, A\&A, 465, 711

Gabor, J. M., \& Bournaud, F. 2013, MNRAS, 434, 606

Gallazzi, A., Bell, E. F., Zibetti, S., Brinchmann, J., \& Kelson, D. D. 2014, ApJ, 788, 72

Genel, S., Nelson, D., Pillepich, A., et al. 2018, MNRAS, 474, 3976

Genzel, R., Tacconi, L. J., Lutz, D., et al. 2015, ApJ, 800, 20

Goddard, D., Thomas, D., Maraston, C., et al. 2017, MNRAS, 466, 4731

González Delgado, R. M., García-Benito, R., Pérez, E., et al. 2015, A\&A, 581, A103

Gunn, J. E., Gott, J., \& Richard, I. 1972, ApJ, 176, 1

Haines, C. P., Iovino, A., Krywult, J., et al. 2017, A\&A, 605, A4

Hopkins, P. F., Bundy, K., Croton, D., et al. 2010, ApJ, 715, 202

Hopkins, P. F., Cox, T. J., Hernquist, L., et al. 2013, MNRAS, 430, 1901

Hopkins, P. F., Hernquist, L., Cox, T. J., et al. 2006, ApJS, 163, 1

Hopkins, P. F., Hernquist, L., Cox, T. J., \& Kereš, D. 2008, ApJS, 175, 356
Huang, S., Haynes, M. P., Giovanelli, R., \& Brinchmann, J. 2012, ApJ, 756,113

Jørgensen, I., Chiboucas, K., Berkson, E., et al. 2017, AJ, 154, 251

Kauffmann, G., Heckman, T. M., White, S. D. M., et al. 2003, MNRAS, 341,33

Kaviraj, S., Kirkby, L. A., Silk, J., \& Sarzi, M. 2007, MNRAS, 382, 960

Keating, S. K., Abraham, R. G., Schiavon, R., et al. 2015, ApJ, 798, 26

Kereš, D., Katz, N., Weinberg, D. H., \& Davé, R. 2005, MNRAS, 363, 2

Kriek, M., Labbé, I., Conroy, C., et al. 2010, ApJL, 722, L64

Kriek, M., van Dokkum, P. G., Labbé, I., et al. 2009, ApJ, 700, 221

Lang, P., Wuyts, S., Somerville, R. S., et al. 2014, ApJ, 788, 11

Le Borgne, D., Abraham, R., Daniel, K., et al. 2006, ApJ, 642, 48

Le Fèvre, O., Saisse, M., Mancini, D., et al. 2003, Proc. SPIE, 4841, 1670

Lemaux, B. C., Lubin, L. M., Shapley, A., et al. 2010, ApJ, 716, 970

Lemaux, B. C., Tomczak, A. R., Lubin, L. M., et al. 2017, MNRAS, 472, 419

Leroy, A. K., Walter, F., Brinks, E., et al. 2008, AJ, 136, 2782

Li, H., Mao, S., Cappellari, M., et al. 2018, MNRAS, in press (arXiv:1802. 01819)

Lilly, S. J., \& Carollo, C. M. 2016, ApJ, 833, 1

Lotz, J. M., Jonsson, P., Cox, T. J., et al. 2011, ApJ, 742, 103

Maltby, D. T., Almaini, O., Wild, V., et al. 2018, MNRAS, 480, 381

Man, A. W. S., Zirm, A. W., \& Toft, S. 2016, ApJ, 830, 89

Marques-Chaves, R., Pérez-Fournon, I., Gavazzi, R., et al. 2018, ApJ, 854, 151

McDermid, R. M., Alatalo, K., Blitz, L., et al. 2015, MNRAS, 448, 3484

Mihos, J. C., \& Hernquist, L. 1994, ApJL, 437, L47

Mihos, J. C., McGaugh, S. S., \& de Blok, W. J. G. 1997, ApJL, 477, L79

Momcheva, I. G., van Dokkum, P. G., van der Wel, A., et al. 2017, PASP, 129, 015004

Moresco, M., Pozzetti, L., Cimatti, A., et al. 2013, A\&A, 558, A61

Mosleh, M., Tacchella, S., Renzini, A., et al. 2017, ApJ, 837, 2

Muzzin, A., Marchesini, D., Stefanon, M., et al. 2013, ApJ, 777, 18

Muzzin, A., van der Burg, R. F. J., McGee, S. L., et al. 2014, ApJ, 796, 65

Muzzin, A., Wilson, G., Yee, H. K. C., et al. 2012, ApJ, 746, 188

Paccagnella, A., Vulcani, B., Poggianti, B. M., et al. 2018, MNRAS, 482, 881

Paulino-Afonso, A., Sobral, D., Buitrago, F., \& Afonso, J. 2017, MNRAS, 465, 2717

Peng, C. Y., Ho, L. C., Impey, C. D., \& Rix, H.-W. 2010, AJ, 139, 2097

Pillepich, A., Springel, V., Nelson, D., et al. 2018, MNRAS, 473, 4077

Poggianti, B. M., Aragón-Salamanca, A., Zaritsky, D., et al. 2009, ApJ, 693, 112

Poggianti, B. M., Calvi, R., Bindoni, D., et al. 2013, ApJ, 762, 77

Popping, G., Caputi, K. I., Trager, S. C., et al. 2015, MNRAS, 454, 2258

Popping, G., Decarli, R., Man, A. W. S., et al. 2017, A\&A, 602, A11

Rodriguez-Gomez, V., Genel, S., Vogelsberger, M., et al. 2015, MNRAS, 449,49

Saintonge, A., Kauffmann, G., Wang, J., et al. 2011, MNRAS, 415, 61

Schawinski, K., Urry, C. M., Simmons, B. D., et al. 2014, MNRAS, 440, 889

Schiminovich, D., Catinella, B., Kauffmann, G., et al. 2010, MNRAS, 408, 919

Schruba, A., Leroy, A. K., Walter, F., et al. 2011, AJ, 142, 37

Scott, N., Brough, S., Croom, S. M., et al. 2017, MNRAS, 472, 2833

Scoville, N., Abraham, R. G., Aussel, H., et al. 2007, ApJS, 172, 38

Shankar, F., Marulli, F., Bernardi, M., et al. 2010, MNRAS, 403, 117

Shen, S., Mo, H. J., White, S. D. M., et al. 2003, MNRAS, 343, 978

Sijacki, D., Springel, V., Di Matteo, T., \& Hernquist, L. 2007, MNRAS, 380,877

Siudek, M., Małek, K., Scodeggio, M., et al. 2017, A\&A, 597, A107

Smercina, A., Smith, J. D. T., Dale, D. A., et al. 2018, ApJ, 855, 51

Snyder, G. F., Cox, T. J., Hayward, C. C., Hernquist, L., \& Jonsson, P. 2011, ApJ, 741, 77

Sobral, D., Best, P. N., Smail, I., et al. 2011, MNRAS, 411, 675

Sommariva, V., Mannucci, F., Cresci, G., et al. 2012, A\&A, 539, A136

Springel, V., Di Matteo, T., \& Hernquist, L. 2005, ApJL, 620, L79

Straatman, C. M. S., van der Wel, A., Bezanson, R., et al. 2018, ApJ, in press (arXiv:1809.08236)

Strateva, I., Ivezić, Ž., Knapp, G. R., et al. 2001, AJ, 122, 1861

Szomoru, D., Franx, M., Bouwens, R. J., et al. 2011, ApJL, 735, L22

Szomoru, D., Franx, M., van Dokkum, P. G., et al. 2013, ApJ, 763, 73

Tacchella, S., Carollo, C. M., Förster Schreiber, N. M., et al. 2018, ApJ, 859,56

Tacchella, S., Carollo, C. M., Renzini, A., et al. 2015, Sci, 348, 314

Tacchella, S., Dekel, A., Carollo, C. M., et al. 2016, MNRAS, 458, 242

Tacconi, L. J., Genzel, R., Saintonge, A., et al. 2018, ApJ, 853, 179

Tacconi, L. J., Neri, R., Genzel, R., et al. 2013, ApJ, 768, 74

Terrazas, B. A., Bell, E. F., Henriques, B. M. B., et al. 2016, ApJL, 830, L12

Terrazas, B. A., Bell, E. F., Woo, J., \& Henriques, B. M. B. 2017, ApJ, 844,170 
Thomas, D., Maraston, C., \& Johansson, J. 2011, MNRAS, 412, 2183

Tran, K.-V. H., Franx, M., Illingworth, G., Kelson, D. D., \& van Dokkum, P. 2003, ApJ, 599, 865

Tran, K.-V. H., Franx, M., Illingworth, G. D., et al. 2004, ApJ, 609, 683

Trujillo, I., Conselice, C. J., Bundy, K., et al. 2007, MNRAS, 382, 109

Trujillo, I., Ferreras, I., \& de La Rosa, I. G. 2011, MNRAS, 415, 3903

van der Wel, A., Bell, E. F., Häussler, B., et al. 2012, ApJS, 203, 24

van der Wel, A., Bell, E. F., van den Bosch, F. C., Gallazzi, A., \& Rix, H.-W. 2009, ApJ, 698, 1232

van der Wel, A., Franx, M., van Dokkum, P. G., et al. 2014, ApJ, 788, 28

van der Wel, A., Noeske, K., Bezanson, R., et al. 2016, ApJS, 223, 29

van Dokkum, P. G., \& Franx, M. 2001, ApJ, 553, 90

Vergani, D., Scodeggio, M., Pozzetti, L., et al. 2008, A\&A, 487, 89

Vergani, D., Zamorani, G., Lilly, S., et al. 2010, A\&A, 509, A42

Wang, E., Li, C., Xiao, T., et al. 2018, ApJ, 856, 137

Wellons, S., Torrey, P., Ma, C.-P., et al. 2015, MNRAS, 449, 361

Whitaker, K. E., van Dokkum, P. G., Brammer, G., \& Franx, M. 2012, ApJL, 754, L29

Wild, V., Almaini, O., Dunlop, J., et al. 2016, MNRAS, 463, 832
Wild, V., Walcher, C. J., Johansson, P. H., et al. 2009, MNRAS, 395, 144

Williams, C. C., Giavalisco, M., Bezanson, R., et al. 2017, ApJ, 838, 94

Williams, R. J., Quadri, R. F., Franx, M., et al. 2010, ApJ, 713, 738

Willmer, C. N. A., Faber, S. M., Koo, D. C., et al. 2006, ApJ, 647, 853

Worthey, G., \& Ottaviani, D. L. 1997, ApJS, 111, 377

Wu, P.-F. 2018, MNRAS, 473, 5468

Wu, P.-F., Gal, R. R., Lemaux, B. C., et al. 2014, ApJ, 792, 16

Wu, P.-F., Kudritzki, R.-P., Tully, R. B., \& Neill, J. D. 2015, ApJ, 810, 151

Wu, P.-F., van der Wel, A., Gallazzi, A., et al. 2018, ApJ, 855, 85

Xu, C. K., Zhao, Y., Scoville, N., et al. 2012, ApJ, 747, 85

Yan, R., Newman, J. A., Faber, S. M., et al. 2006, ApJ, 648, 281

Yan, R., Newman, J. A., Faber, S. M., et al. 2009, MNRAS, 398, 735

Yang, Y., Zabludoff, A. I., Zaritsky, D., \& Mihos, J. C. 2008, ApJ, 688, 945

Yano, M., Kriek, M., van der Wel, A., \& Whitaker, K. E. 2016, ApJL, $817, \mathrm{~L} 21$

Yesuf, H. M., Faber, S. M., Trump, J. R., et al. 2014, ApJ, 792, 84

Zahid, H. J., \& Geller, M. J. 2017, ApJ, 841, 32

Zanella, A., Scarlata, C., Corsini, E. M., et al. 2016, ApJ, 824, 68

Zolotov, A., Dekel, A., Mandelker, N., et al. 2015, MNRAS, 450, 2327 